TRANSACTIONS OF THE

AMERICAN MATHEMATICAL SOCIETY

Volume 364, Number 10, October 2012, Pages 5173-5208

S 0002-9947(2012)05448-1

Article electronically published on May 15, 2012

\title{
CHARACTER VARIETIES
}

\author{
ADAM S. SIKORA
}

ABSTRACT. We study properties of irreducible and completely reducible representations of finitely generated groups $\Gamma$ into reductive algebraic groups $G$. In particular, we study the geometric invariant theory of the $G$ action on the space of $G$-representations of $\Gamma$ by conjugation.

Let $X_{G}(\Gamma)$ be the $G$-character variety of $\Gamma$. We prove that for every completely reducible, scheme smooth $\rho: \Gamma \rightarrow G$

$$
T_{[\rho]} X_{G}(\Gamma) \simeq T_{0}\left(H^{1}(\Gamma, A d \rho) / / S_{\Gamma}\right),
$$

where $H^{1}(\Gamma, A d \rho)$ is the first cohomology group of $\Gamma$ with coefficients in the Lie algebra $\mathfrak{g}$ of $G$ twisted by $\Gamma \stackrel{\rho}{\longrightarrow} G \stackrel{A d}{\longrightarrow} G L(\mathfrak{g})$ and $S_{\Gamma}$ is the centralizer of $\rho(\Gamma)$ in $G$. The condition of $\rho$ being scheme smooth is very important as there are groups $\Gamma$ such that

$$
\operatorname{dim} T_{[\rho]} X_{G}(\Gamma)<T_{0} H^{1}(\Gamma, A d \rho),
$$

for a Zariski open subset of points in $X_{G}(\Gamma)$. We prove, however, that all irreducible representations of surface groups are scheme smooth.

Let $M$ be an orientable 3-manifold with a connected boundary $F$ of genus $g \geq 2$. Let $X_{G}^{g}(F)$ be the subset of the $G$-character variety of $\pi_{1}(F)$ composed of conjugacy classes of good representations $\rho: \Gamma \rightarrow G$, i.e., irreducible representations such that the centralizer of $\rho(\Gamma)$ is the center of $G$. By a theorem of Goldman, $X_{G}^{g}(F)$ is a holomorphic symplectic manifold. We prove that the set of good $G$-representations of $\pi_{1}(F)$ which extend to representations of $\pi_{1}(M)$ is a complex isotropic subspace of $X_{G}^{g}(F)$. It is Lagrangian, if these representations correspond to reduced points of the $G$-character variety of $M$. It is an open problem whether it is always the case.

\section{Contents}

1. Summary of results $\quad 5174$

2. Reductive groups $\quad 5176$

3. Irreducible and completely reducible subgroups $\quad 5177$

4. Stabilizers of irreducible representations $\quad 5179$

5. Representation varieties $\quad 5181$

6. Spaces of irreducible representations 5183

7. Stable and properly stable representations in the sense of GIT 5184

8. Tangent spaces 5

9. Smooth and reduced representations 5187

10. Orbits $\quad 5189$

11. Character varieties 5191

Received by the editors January 31, 2010 and, in revised form, May 25, 2010, and August 23, 2010.

2010 Mathematics Subject Classification. Primary 14D20; Secondary 14L24, 57M27.

Key words and phrases. Representation variety, character variety, irreducible representation, completely reducible representation, Goldman symplectic form, 3-manifold, Lagrangian submanifold.

(C)2012 American Mathematical Society Reverts to public domain 28 years from publication 
12. Character varieties as algebraic schemes 5193

13. Tangent spaces to character varieties 5194

14. Symplecticity of character varieties of surfaces 5197

15. 3-manifolds and Lagrangian subspaces $\quad 5199$

References 5201

\section{Summary OF RESUlts}

Let $G$ be a complex reductive algebraic group, for example a classical group of matrices, $G L(n, \mathbb{C}), S L(n, \mathbb{C}), O(n, \mathbb{C}), S p(n, \mathbb{C})$, or its quotient. Let $\Gamma$ be a finitely generated group. We say that a representation $\rho: \Gamma \rightarrow G$ is irreducible if $\rho(\Gamma)$ is not contained in any proper parabolic subgroup of $G$. Additionally, we say that $\rho$ is completely reducible if for every parabolic subgroup $P \subset G$ containing $\rho(\Gamma)$, there is a Levi subgroup $L \subset P$ containing $\rho(\Gamma)$. In particular, $\rho: \Gamma \rightarrow G L(n, \mathbb{C})$ is irreducible if and only if $\mathbb{C}^{n}$ is a simple $\Gamma$-module (via $\rho$ ), and it is completely reducible if $\mathbb{C}^{n}$ is a semi-simple $\Gamma$-module.

We discuss properties of irreducible and completely reducible representations in Sections 34, For example, we prove that $\rho: \Gamma \rightarrow G$ is completely reducible if and only if the algebraic closure of $\rho(\Gamma)$ in $G$ is a linearly reductive group. Furthermore, a completely reducible representation $\rho: \Gamma \rightarrow G$ is irreducible if and only if the centralizer of $\rho(\Gamma)$ is a finite extension of $C(G)$.

The space, $\operatorname{Hom}(\Gamma, G)$, of all group homomorphisms from $\Gamma$ to $G$ is an algebraic set on which $G$ acts by conjugation. In Section 7 we study properties of this action from the point of view of the Geometric Invariant Theory. In particular, we observe that $\rho$ is a poly-stable point under that action if and only if $\rho$ is completely reducible. If $\rho$ is irreducible, then it is a stable point. Finally, $\rho$ is properly stable if and only if $\rho$ is irreducible and $C(G)$ is finite.

The categorical quotient, $X_{G}(\Gamma)=\operatorname{Hom}(\Gamma, G) / / G$, is called the $G$-character variety of $\Gamma$, cf. Section 11. Although it is a coarser quotient than the set theory one, it has the advantage of having a natural structure of an affine algebraic set. Every element of $X_{G}(\Gamma)$ is represented by a unique completely reducible representation. With $\operatorname{Hom}(\Gamma, G)$ and $X_{G}(\Gamma)$, there are naturally associated algebraic schemes $\mathcal{H o m}(\Gamma, G)$ and $\mathcal{X}_{G}(\Gamma)=\mathcal{H o m}(\Gamma, G) / / G$ such that $\mathbb{C}[H o m(\Gamma, G)]$ and $\mathbb{C}\left[X_{G}(\Gamma)\right]$ are nil-radical quotients of $\mathcal{O}(\mathcal{H o m}(\Gamma, G))$ and $\mathcal{O}\left(\mathcal{X}_{G}(\Gamma)\right)$, cf. Sections 5 and 12 .

We formulate cohomological descriptions of the tangent spaces to $\mathcal{X}_{G}(\Gamma)$ and to $X_{G}(\Gamma)$ in Section 13 . More specifically, we prove the following: Let $H^{*}(\Gamma, A d, \rho)$ denote the group cohomology of $\Gamma$ with coefficients in the Lie algebra $\mathfrak{g}$ of $G$ twisted by the homomorphism

$$
\Gamma \stackrel{\rho}{\longrightarrow} G \stackrel{A d}{\longrightarrow} \operatorname{End}(\mathfrak{g}),
$$

where $A d$ is the adjoint representation. Denote the stabilizer of $\rho$ under the $G$ action (by conjugation) on $\operatorname{Hom}(\Gamma, G)$ by $S_{\rho}$. It is the centralizer of $\rho(\Gamma)$ in $G$. There is a natural action of $S_{\rho}$ on $H^{1}(\Gamma, A d \rho)$, cf. Section 13 .

We call an irreducible $\rho: \Gamma \rightarrow G$ good if the stabilizer of its image, $S_{\rho}$, is the center of $G$. We say that $\rho$ is scheme smooth if $\mathcal{H o m}(\Gamma, G)$ is non-singular at $\rho$. 
Theorem 1 (cf. Theorem 53). (1) For every good $\rho: \Gamma \rightarrow G$ there exists a natural linear isomorphism

$$
\phi: H^{1}(\Gamma, A d \rho) \stackrel{\cong}{\rightrightarrows} T_{[\rho]} \mathcal{X}_{G}(\Gamma) .
$$

(2) If $\rho: \Gamma \rightarrow G$ is completely reducible and scheme smooth, then

$$
T_{0}\left(H^{1}(\Gamma, A d \rho) / / S_{\rho}\right) \simeq T_{[\rho]} \mathcal{X}_{G}(\Gamma)=T_{[\rho]} X_{G}(\Gamma) .
$$

The quotient on the left side in the statement of Theorem 1(2) may be non-trivial even if $\rho$ is irreducible, cf. discussion in Section 4 and Example 20 in particular. We discuss the question of the existence of a natural isomorphism in part (2) of the above theorem in Section 13 ,

Versions of the above theorem for $G=\operatorname{PSL}(2, \mathbb{C})$ appear in $\mathrm{Po}$, Proposition 3.5] and [HP2, Prop. 5.2]. Although statements similar to Theorem 1(1) appear in the literature for general $G$, there is widespread confusion about the necessary assumptions and all proofs known to us are incomplete.

The following result illuminates the importance of the requirement of $\rho$ being scheme smooth in Theorem 1(2). Denote the set of equivalence classes of good representations in $\mathcal{X}_{G}(\Gamma)$ by $\mathcal{X}_{G}^{g}(\Gamma)$.

Theorem 2 (cf. Theorem [57). $\mathcal{X}_{G}^{g}(\Gamma)$ is reduced iff $T_{[\rho]} X_{G}(\Gamma)=H^{1}(\Gamma, A d \rho)$ for a non-empty Zariski open set of $[\rho]$ 's in $X_{G}^{g}(\Gamma)$.

It is easy to see that all representations of free groups are scheme smooth. We prove an analogous statement for surface groups, i.e., fundamental groups of closed, orientable surfaces of genus $\geq 2$.

Theorem 3. For every reductive $G$, all irreducible $G$-representations of surface groups are scheme smooth.

This theorem has some important consequences to the theory of skein modules, cf. Corollary 52 :

Theorem 4. For every orientable surface $F$, there is an isomorphism between the Kauffman bracket skein algebra $\mathcal{S}_{2, \infty}(F, \mathbb{C},-1)$ and $\mathbb{C}\left[X_{S L(2, \mathbb{C})}\left(\pi_{1}(F)\right)\right]$ sending a link $K_{1} \cup \ldots \cup K_{n}$ in $F \times[0,1]$ to $(-1)^{n} \tau_{K_{1}} \cdot \ldots \cdot \tau_{K_{n}}$, where $\tau_{K} \in \mathbb{C}\left[X_{S L(2, \mathbb{C})}\left(\pi_{1}(F)\right)\right]$ sends the equivalence class of $\rho: \pi_{1}(F) \rightarrow S L(2, \mathbb{C})$ to $\operatorname{tr} \rho(\gamma)$, and $\gamma \in \pi_{1}(F)$ is any element representing $K$ (with some orientation).

Although this result was announced in [PS2, Thm. 7.3], its proof relied on [PS2, Thm. 7.4] whose proof was not provided. An alternative proof of the above statement was provided independently by L. Charles and J. Marché in [CM].

Denote the set of irreducible $G$-representations of $\Gamma$ by $\operatorname{Hom}^{i}(\Gamma, G)$. It is a Zariski open subset of $\operatorname{Hom}(\Gamma, G)$, cf. Proposition 27. Since each equivalence class in $\operatorname{Hom}(\Gamma, G) / / G$ contains a unique closed orbit and the orbit of every irreducible representation is closed (Theorem 30), the categorical quotient of $\operatorname{Hom}(\Gamma, G)$ restricted to $\operatorname{Hom}^{i}(\Gamma, G)$ is the set theoretic quotient. Denote $\operatorname{Hom}^{i}(\Gamma, G) / G$ by $X_{G}^{i}(\Gamma)$.

Proposition 5. Let $\Gamma$ be a free group or a surface group. Then

(1) $X_{G}^{i}(\Gamma)$ is an orbifold.

(2) If $G=G L(n, \mathbb{C})$ or $S L(n, \mathbb{C})$, then $X_{G}^{i}(\Gamma)$ is a manifold. (See also [FL2.) 
We do not know if Proposition 5(2) holds for any reductive groups other than $G L(n, \mathbb{C})$ and $S L(n, \mathbb{C})$, cf. Question 19 and Proposition 49 .

We say that $\rho$ is good if $\rho$ is irreducible and $S_{\rho}$ is the center of $G$. Denote the set of all such representations by $\operatorname{Hom}^{g}(\Gamma, G)$. It is a Zariski open subset of $\operatorname{Hom}(\Gamma, G)$, cf. Proposition 33. Let

$$
X_{G}^{g}(\Gamma)=H_{o m}^{g}(\Gamma, G) / / G=H_{o m}^{g}(\Gamma, G) / G .
$$

By the above discussion $X_{G}^{g}(\Gamma)$ is an open subset of $X_{G}^{i}(\Gamma)$. Furthermore, it is a smooth manifold for free groups and surface groups $\Gamma$.

For a topological space $Y$, we abbreviate $X_{G}\left(\pi_{1}(Y)\right)$ by $X_{G}(Y)$. Let $F$ be a closed orientable surface of genus $\geq 2$. Goldman proved that every non-degenerate symmetric bilinear $A d$-invariant form $B$ on $\mathfrak{g}$ gives rise to a holomorphic symplectic 2 -form $\omega_{B}$ on $X_{G}^{g}(F)$, Go2], cf. Section 14.

According to folk knowledge, if $M$ is a compact orientable 3-manifold with a connected boundary $F$, then the image of the map $r^{*}: X_{G}(M) \rightarrow X_{G}(F)$ induced by the embedding $r: F=\partial M \hookrightarrow M$ is "roughly speaking" a Lagrangian subspace of the $G$-character variety of $F$. In Section [15] we formulate this claim precisely, and we prove it in detail. Let $Y_{G}(M)$ be the non-singular part of

$$
X_{G}^{g}(F) \cap r^{*}\left(X_{G}(M)\right) .
$$

Theorem 6. (1) $Y_{G}(M)$ is an isotropic submanifold of $X_{G}^{g}(F)$ with respect to $\omega_{B}$.

In particular, every connected component of $Y_{G}(M)$ of dimension $\frac{1}{2} \operatorname{dim} X_{G}(F)$ is Lagrangian.

(2) If a connected component $C$ of $Y_{G}(M)$ contains the conjugacy class of a representation which is a reduced point of $\mathcal{H o m}\left(\pi_{1}(M), G\right)$, then $C$ is a Lagrangian submanifold of $Y_{G}(M)$.

Although we do not know any example of a 3-manifold $M$ such that $Y_{G}(M)$ is not Lagrangian, in light of the above theorem and the results of M. Kapovich mentioned in Section 12, we believe that such examples exist.

Here is a different version of (2) above:

Theorem 7. If $X_{G}^{s}(M)$ denotes the set of equivalence classes of scheme smooth representations in $X_{G}(M)$, then $X_{G}^{g}(F) \cap r^{*}\left(X_{G}^{s}(M)\right)$ is an immersed Lagrangian submanifold of $X_{G}^{g}(F)$.

Note however that $X_{G}^{s}(M)$ may be empty even if $\pi_{1}(M)$ has good $G$-representations.

Theorems 6 and 7 are relevant to Chern-Simons theory, cf. Section 15 .

In this paper we assume familiarity with basic algebraic geometry and the theory of algebraic groups. The standard references for these topics are [ $\mathrm{Ha}, \mathrm{Shf}, \mathrm{Bo}, \mathrm{Hu}$.

We would like to thank S. Baseilhac, H. Boden, F. Bonahon, W. Goldman, C. Frohman, M. Kapovich, S. Lawton, J. Porti, and the referee for helpful comments.

\section{ReduCtive Groups}

Every algebraic group $G$ contains a unique maximal normal connected solvable subgroup called its radical and is denoted by $\operatorname{Rad} G$. A connected group $G$ is semisimple if and only if $\operatorname{Rad} G$ is trivial. A connected group $G$ is reductive if and only if $\operatorname{Rad} G$ is an algebraic torus, $\left(\mathbb{C}^{*}\right)^{n}$. In particular, $\mathbb{C}^{*}$ and all classical matrix 
groups, $S L(n, \mathbb{C}), O(n, \mathbb{C}), S p(n, \mathbb{C})$, are reductive. Furthermore, Cartesian products, quotients and finite connected covers of reductive groups are reductive. In fact, all reductive groups can be obtained in this way from simple algebraic groups.

Denote the center of $G$ by $C(G)$ and the connected component of the identity in $C(G)$ by $C^{0}(G)$. For every reductive $G, C^{0}(G)=\left(\mathbb{C}^{*}\right)^{n}$. A reductive group $G$ is semi-simple if and only if $C^{0}(G)$ is trivial.

Let $[G, G]$ be the commutator of $G$. If $G$ is reductive, then $[G, G]$ is semi-simple. Furthermore, by [Bo, Proposition IV.14.2], the epimorphism

$$
\nu: C^{0}(G) \times[G, G] \rightarrow G, \quad \nu(g, h)=g \cdot h
$$

has a finite kernel isomorphic to

$$
K=C^{0}(G) \cap[G, G] .
$$

Therefore, there is a finite quotient

$$
\pi: G \rightarrow C^{0}(G) / K \times[G, G] / K .
$$

An algebraic group $G$ is linearly reductive if all its $G L(n, \mathbb{C})$-representations are completely reducible. $G$ is linearly reductive if and only if the connected component, $G^{0}$, of its identity is reductive. (This property does not hold for groups over fields of non-zero characteristic.) Therefore, linearly reductive groups are "virtually reductive".

A maximal connected solvable subgroup of $G$ is called a Borel subgroup. A closed subgroup $P \subset G$ is parabolic if one of the following equivalent conditions holds:

(a) $G / P$ is a complete variety,

(b) $G / P$ is a projective variety,

(c) $P$ contains a Borel subgroup of $G$, cf. $\mathrm{BO}$.

A Levi subgroup of an algebraic group $H$ is a connected subgroup $L \subset H$ such that $H$ is a semi-direct product of $L$ and the unipotent radical of $H$. Since $L$ is isomorphic to the quotient of $H$ by its unipotent radical, it is always reductive. By a result of Mostow, every algebraic group contains a Levi subgroup, cf. Bo, IV.11.22]

\section{IRREDUCIBLE AND COMPLETELY REDUCIBLE SUBGROUPS}

We say that a subgroup $H$ (closed or not) of $G$ is irreducible if it is not contained in any proper parabolic subgroup of $G$. We also say that $H$ is completely reducible if for every parabolic subgroup $P \subset G$ containing $H$, there is a Levi subgroup of $P$ containing $H$ as well, $\mathrm{Se}$, BMR. In particular, every irreducible subgroup is completely reducible.

The following is an important characterization of completely reducible subgroups:

Proposition 8. For every reductive $G$, a subgroup $H \subset G$ is completely reducible if and only if the algebraic closure of $H$ in $G$ is a linearly reductive group.

Proof. $\Rightarrow(1)$ Assume first that $H$ is irreducible, i.e., not contained in any proper parabolic subgroup of $G$. Let $\bar{H}$ be the Zariski closure of $H$ in $G$ and let $\operatorname{Rad}_{u}(\bar{H})$ be the unipotent radical of $\bar{H}$. Let $P=\mathcal{P}\left(\operatorname{Rad}_{u}(\bar{H})\right)$ be the parabolic subgroup defined in [Hu, 30.3]. Then $\bar{H} \subset N_{G}\left(\operatorname{Rad}_{u}(\bar{H})\right)$ and $N_{G}\left(\operatorname{Rad}_{u}(\bar{H})\right) \subset P$ by $[\mathrm{Hu}$, 30.3 Corollary A]. If $\bar{H}$ is not linearly reductive, then $\operatorname{Rad}_{u}(\bar{H})$ is non-trivial and 
$\operatorname{Rad}_{u}(\bar{H}) \subset \operatorname{Rad}_{u}(P)$ by $[\mathrm{Hu}, 30.3$ Corollary A]. Therefore, $P$ is a proper subgroup of $G$.

(2) Now we carry the proof in full generality by induction with respect to $\operatorname{dim} G$ : If $\operatorname{dim} G=1$, then $G=\mathbb{C}^{*}$ and the statement holds. Assume now that it holds for all reductive algebraic groups $G$ of dimension less than $n$. Let $\operatorname{dim} G=n$. If $H$ lies in a proper parabolic subgroup of $G$, then it also lies in a Levi subgroup of $P$ and the statement follows from inductive hypothesis. If $H$ does not lie in a proper parabolic subgroup of $G$, then $H$ is irreducible in $G$ and the statement follows from part (1).

$\Leftarrow$ Suppose $\bar{H}$ is linearly reductive and $H \subset P$. Since $P$ is closed, $\bar{H} \subset P$. Now the statement follows from the fact that every closed linearly reductive subgroup of $P$ lies in a Levi subgroup of $P$. Since we do not know a good reference to this fact, we enclose its proof here: There is an exact sequence

$$
\{e\} \rightarrow \operatorname{Rad}_{U} P \rightarrow P \stackrel{\tau}{\longrightarrow} L \rightarrow\{e\},
$$

where $\operatorname{Rad}_{U} P$ is the unipotent radical of $P$. Since $\bar{H}^{0}$ is reductive, it has no connected unipotent subgroups and, therefore, $\tau$ is an embedding of $\bar{H}^{0}$ into $L$. Therefore, the kernel $K$ of $\tau$ restricted $\bar{H}$ is finite. By [Bo, Corollary 4.8], $\operatorname{Rad}_{U} P$ is a subgroup of upper triangular matrices and, therefore, it has no elements of finite order. Hence, $K$ is trivial.

A representation $\phi: \Gamma \rightarrow G$ is irreducible or completely reducible if $\phi(\Gamma) \subset G$ is. In particular, a representation $\rho: \Gamma \rightarrow G L(n, \mathbb{C})$ is irreducible if and only if $\mathbb{C}^{n}$ does not have any $\Gamma$-invariant subspaces other than $\{0\}$ and $\mathbb{C}^{n}$. Additionally, $\rho: \Gamma \rightarrow G L(n, \mathbb{C})$ is completely reducible if and only if $\mathbb{C}^{n}$ decomposes into a sum of irreducible $\Gamma$-modules.

Since a quotient of a reductive group is reductive, Proposition 8 implies:

Corollary 9. For every homomorphism $\phi: G_{1} \rightarrow G_{2}$ of reductive groups, the image of a completely reducible subgroup of $G_{1}$ is completely reducible in $G_{2}$.

Similarly, we have:

Lemma 10. For every epimorphism $\phi: G_{1} \rightarrow G_{2}$ of reductive groups, the image of an irreducible subgroup of $G_{1}$ is irreducible in $G_{2}$.

Proof. Suppose that $\phi(H)$ lies inside a proper parabolic subgroup $P \subset G_{2}$. Since $\phi$ induces an isomorphism $G_{1} / \phi^{-1}(P) \rightarrow G_{2} / P$ and $G_{2} / P$ is complete, $G_{1} / \phi^{-1}(P)$ is complete as well, implying that $\phi^{-1}(P)$ is a proper parabolic subgroup of $G_{1}$ containing $H$.

The following example shows that irreducibility of $H \subset G_{1}$ does not imply irreducibility of $\phi(H) \subset G_{2}$ if $\phi: G_{1} \rightarrow G_{2}$ is not an epimorphism, even if $\phi$ is irreducible itself.

Proposition 11. Let $H=\left\{A: A \cdot A^{T}= \pm I\right\} \subset S L(2, \mathbb{C})$.

(1) $H$ is isomorphic to $O(2, \mathbb{C})$.

(2) $H \subset S L(2, \mathbb{C})$ is irreducible.

(3) The image of $H$ under the adjoint representation $A d: S L(2, \mathbb{C}) \rightarrow S L(3, \mathbb{C})$ is completely reducible but not irreducible in $S L(3, \mathbb{C})$. 
Proof. (1) $H$ is a non-abelian split $\mathbb{Z} / 2$ extension of $S O(2) \simeq \mathbb{C}^{*}$. However, since $H^{2}\left(\mathbb{Z} / 2, \mathbb{C}^{*}\right)=0$ (with twisted coefficients), $O(2, \mathbb{C})$ is the unique non-abelian split extension of $S O(2, \mathbb{C})$ by $\mathbb{Z} / 2$.

(2) Since $H$ is reductive, it is completely reducible in $S L(2, \mathbb{C})$ by Proposition 8. If it was reducible, it would be a subgroup of diagonal matrices, $\mathbb{C}^{*}$. Since $H$ is non-abelian, it is irreducible in $S L(2, \mathbb{C})$.

(3) Complete reducibility follows from Corollary 9. We claim that the group $A d(H)$ lies in the parabolic subgroup of $S L(3, \mathbb{C})$ composed of transformations of $\operatorname{sl}(2, \mathbb{C}) \simeq \mathbb{C}^{3}$ which preserve $\operatorname{Span}(M) \subset \operatorname{sl}(2, \mathbb{C})$, where $M=\left(\begin{array}{cc}0 & -1 \\ 1 & 0\end{array}\right)$. Indeed, since $S L(2, \mathbb{C})=S p(2, \mathbb{C}), A M A^{T}=M$ for every $A \in S L(2, \mathbb{C})$. If $A \in H$, then $A^{T}= \pm A^{-1}$, and the claim follows.

We say that $H \subset G$ is Ad-irreducible if $A d(H) \subset G L(\mathfrak{g})$ is irreducible. The $H \subset S L(2, \mathbb{C})$ above is irreducible but not $A d$-irreducible. By Proposition 8 , every irreducible subgroup $H \subset G$ is completely Ad-reducible, i.e., $\operatorname{Ad}(H) \subset G L(\mathfrak{g})$ is completely reducible. We are going to show that $A d$-irreducibility implies irreducibility.

Lemma 12. Let $\phi: G \rightarrow G L(n, \mathbb{C})$ be an irreducible representation of a reductive group $G$. If $H$ a subgroup of $G$ such that $\phi(H)$ is irreducible, then either

(a) $H$ is irreducible, or

(b) Ker $\phi$ contains the unipotent radical (i.e., the maximal connected unipotent subgroup) of a Borel subgroup of $G$.

Proof. Suppose that $H \subset P \subsetneq G$. Then $\phi$ restricted to $P$ is irreducible as well. Let $U$ be the unipotent radical of $P$. Denote the space of vectors in $V=\mathbb{C}^{n}$ invariant under the $U$-action by $V^{U}$. Since $P$ is a semi-direct product of $U$ and its Levi subgroup $L$, [Bo], $P=L \rtimes U, l^{-1} u l \in U$, for every $u \in U$ and $l \in L$, and

$$
u \cdot l \cdot v=l \cdot l^{-1} u l \cdot v=l \cdot v \quad \text { for every } v \in V^{U} .
$$

Therefore $l \cdot v \in V^{U}$ and, consequently, $V^{U}$ is preserved by $P$. Since $\phi$ restricted to $P$ is irreducible, by Shur's lemma $V^{U}$ is either 0 or $V$. However, $U$ is a connected solvable group and, therefore, $V^{U} \neq 0$, by the Lie-Kolchin theorem, [Bo, Cor. 10.5]. Hence $V^{U}=V$ and, consequently, $U \subset \operatorname{Ker} \phi$. If $B$ is a Borel subgroup of $G$ contained in $P$, then the unipotent radical of $B$ is contained in $U$.

Since the kernel of the adjoint representation is the center of $G$, [Bo, I.3.15], and its unipotent radical is trivial, Lemma 12 implies:

Corollary 13. Every Ad-irreducible subgroup of a reductive group is irreducible.

\section{Stabilizers of IRREDUCIBLE REPRESENTATIONS}

Proposition 14. The centralizer of an Ad-irreducible subgroup of a reductive group $G$ is the center of $G$.

Proof. Let $H \subset G$ be $A d$-irreducible. By Shur's lemma the centralizer of $A d(H)$ is the group of scalar matrices in $G L(\mathfrak{g})$. Hence,

$$
\operatorname{Ad}\left(C_{G}(H)\right) \subset C_{G L(\mathfrak{g})}(\operatorname{Ad}(H)) \subset\left\{c \cdot I: c \in \mathbb{C}^{*}\right\} .
$$


On the other hand, since the center of $A d(G)=G / C(G)$ is trivial, cf. [FH, Thm. 23.16], $\operatorname{Ad}(G) \cap\left\{c \cdot I: c \in \mathbb{C}^{*}\right\}=\{I\}$. Hence, $\operatorname{Ad}\left(C_{G}(H)\right)$ is trivial, implying that $C_{G}(H) \subset C(G)$.

Proposition 15. The centralizer of an irreducible subgroup of a reductive group $G$ is a finite extension of the center of $G$.

Proof. Suppose that the centralizer, $C_{G}(H)$, of $H$ is an infinite extension of the center. Let $T$ be a maximal torus in $C_{G}(H)$. Then $\operatorname{rank} T>\operatorname{rank} C(G)$ and $H \subset C_{G}(T)$. Recall that $T$ is either regular, semi-regular, or singular, BBo, $\left.\S 13.1\right]$. If $T$ is regular, then $C_{G}(T)$ is a maximal torus. If $T$ is semi-regular, then $C_{G}(T)$ is contained in a Borel subgroup, cf. proof of [Bo, IV.13.1 Proposition]. In either case $H \subset C_{G}(T)$ is reducible. Therefore, $T$ is singular. In that case $T$ is the connected component of identity of $\bigcap_{\alpha \in I} \operatorname{Ker} \alpha$, where the intersection is over a certain proper, non-empty subset $I$ of positive roots. By [Bo, IV.14.17], $T$ lies inside of a proper parabolic subgroup of $G$ (denoted by Borel by $P_{I}$ ).

The following lemma will be useful later.

Lemma 16. For every Levi subgroup $L$ of every proper parabolic subgroup of a reductive group $G, \operatorname{dim} C(L)>\operatorname{dim} C(G)$.

Proof. Suppose that $L$ is a Levi subgroup of a parabolic group $P$ in $G$. As before, let $C^{0}(G)$ be the connected component of the identity in the center of $G$. Then $C^{0}(G) \subset L$ and $L^{\prime}=L / C^{0}(G)$ is a Levi subgroup of the parabolic subgroup $P^{\prime}=P / C^{0}(G)$ of $G^{\prime}=G / C^{0}(G)$. Therefore, without loss of generality we can assume that $\operatorname{dim} C(G)=0$.

By [Bo, Prop. 11.23], it is enough to prove that the radical of $P, \mathcal{R} P$, has a positive dimension. Fix a root system for $G$. We are going to use the notation of [Bo]. By classification of parabolic subgroups in [Bo, $\S 14.17], P=P_{I}$ for some subset $I$ of positive roots $\Delta$ of $G$. Let $T_{I}$ be the identity component of $\bigcap_{\alpha \in I} \operatorname{Ker} \alpha$. By [Bo, Prop. 14.18], $T_{I} \subset \mathcal{R} P$. Since $T_{I}$ is an algebraic torus of dimension rank $G-|I|$, $\operatorname{dim} \mathcal{R} P>0$ unless $I=\Delta$. In this case, $P=G$.

Proposition 15 and Lemma 16 imply:

Corollary 17. A completely reducible subgroup $H \subset G$ is irreducible if and only if $\operatorname{dim} C_{G}(H)=\operatorname{dim} C(G)$.

We will say that a reductive group $G$ has property CI if the centralizer of every irreducible subgroup of $G$ coincides with the center of $G$.

Example 18. $G=G L(n, \mathbb{C})$ and $S L(n, \mathbb{C})$ are CI. Indeed, $H \subset G$ is irreducible if and only if elements of $H$ linearly span $M(n, \mathbb{C})$. Consequently, the centralizer of every irreducible subgroup $H \subset G$ is the center of $G$.

Question 19. Are $G L(n, \mathbb{C})$ and $S L(n, \mathbb{C})$ the only CI-groups?

Example 20. $P S L(2, \mathbb{C})$ is not CI. To see that consider the subgroup $H \subset$ $P S L(2, \mathbb{C})$, generated by $\pm\left(\begin{array}{cc}i & 0 \\ 0 & -i\end{array}\right)$ and $\pm\left(\begin{array}{cc}0 & 1 \\ -1 & 0\end{array}\right)$. Since $|H|=4, H$ is the Klein group. One can easily see that $H$ is its own centralizer in $\operatorname{PSL}(2, \mathbb{C})$ (while the center of $P S L(2, \mathbb{C})$ is trivial). Being finite, $H$ is linearly reductive and completely reducible by Proposition 8. By Corollary 17, $H$ is irreducible in $P S L(2, \mathbb{C})$. 
Example 21. $S O(n, \mathbb{C})$ is not $\mathrm{CI}$ : Let $D M_{n}$ be the group of diagonal matrices in

$$
S O(n, \mathbb{C})=\left\{A: A \cdot A^{T}=I\right\} \subset S L(n, \mathbb{C}) .
$$

Then $D M_{n} \simeq(\mathbb{Z} / 2)^{n-1}$ and it is easy to see that $D M_{n}$ is its own centralizer in $S O(n, \mathbb{C})$. Being finite, $D M_{n}$ is linearly reductive and completely reducible by Proposition 8. By Corollary 17, $D M_{n}$ is irreducible in $S O(n, \mathbb{C})$.

Proposition 22. $S p(2 n, \mathbb{C})$ is not $C I$.

Proof. (Based on the idea of S. Lawton, cf. [FL2]) Denote by $D\left(\alpha_{1}, \ldots, \alpha_{n}\right)$ the diagonal matrix with entries $\alpha_{1}, \ldots, \alpha_{n}$, and by $A D\left(\alpha_{1}, \ldots \alpha_{n}\right)$ the anti-diagonal matrix

$$
\left(\begin{array}{cccc}
0 & 0 & 0 & \alpha_{1} \\
0 & 0 & \ldots & 0 \\
0 & \ldots & 0 & 0 \\
\alpha_{n} & 0 & 0 & 0
\end{array}\right) .
$$

The matrices $D\left(\alpha_{1}, \ldots, \alpha_{n}, \alpha_{n}^{-1}, \ldots, \alpha_{1}^{-1}\right)$ and $A D\left(\beta_{1}, \ldots, \beta_{n},-\beta_{n}^{-1}, \ldots,-\beta_{1}^{-1}\right)$, for $\alpha_{1}, \ldots, \alpha_{n}, \beta_{1}, \ldots, \beta_{n} \in \mathbb{C}^{*}$ form a subgroup of $S p(2 n, \mathbb{C})=\left\{A: A J A^{T}=\right.$ $J\}$, where $J=A D(1, \ldots, 1,-1, \ldots,-1)$. Denote that subgroup by $H_{n}$. An elementary computation shows that the center of $H_{n}$ is composed of matrices $D\left(\alpha_{1}, \ldots, \alpha_{n}, \alpha_{n}^{-1}, \ldots, \alpha_{1}^{-1}\right)$, where $\alpha_{1}, \ldots, \alpha_{n} \in\{ \pm 1\}$. Since $H_{n}$ is a finite extension of $\left(\mathbb{C}^{*}\right)^{n}$, it is linearly reductive and, hence, by Proposition 8 , it is completely reducible in $S p(2 n, \mathbb{C})$. Since $C\left(\Gamma_{n}\right)$ is a finite extension of $C(S p(2 n, \mathbb{C}))=\{ \pm 1\}$, $\Gamma_{n}$ is irreducible by Corollary 17.

By the following result, $P S O(n, \mathbb{C}), P S p(2 n, \mathbb{C})$ are not CI either.

Proposition 23. A quotient of a non-CI group by a finite subgroup is non-CI.

Proof. Let $\Gamma \subset G$ be irreducible and such that $C_{G}(\Gamma)$ is a proper extension of $C(G)$. If $\pi: G \rightarrow G^{\prime}$ is a quotient with finite kernel, then $\operatorname{Ker} \pi \subset C(G)$ and, consequently, the centralizer of $\pi(\Gamma)$ in $G^{\prime}$ is a proper extension of $C\left(G^{\prime}\right)$. Now the statement follows from Lemma 10.

\section{REPRESENTATION VARIETIES}

If $\Gamma$ is a finitely generated group and $G$ an affine complex algebraic group, then the space of all $G$-representations of $\Gamma, \operatorname{Hom}(\Gamma, G)$, is an algebraic set.

Remark 24. $\operatorname{Hom}\left(\Gamma_{1} * \Gamma_{2}, G\right)=\operatorname{Hom}\left(\Gamma_{1}, G\right) \times \operatorname{Hom}\left(\Gamma_{2}, G\right)$. Hence, for the free group on $n$ generators, $\operatorname{Hom}\left(F_{n}, G\right)=G^{n}$.

Example 25. Each point of $\operatorname{Hom}\left(\mathbb{Z}^{2}, S L(2, \mathbb{C})\right)$ is represented by $\rho: \mathbb{Z}^{2} \rightarrow$ $S L(2, \mathbb{C})$ defined by

$$
\rho(1,0)=\left(\begin{array}{ll}
x_{1} & x_{2} \\
x_{3} & x_{4}
\end{array}\right), \quad \rho(0,1)=\left(\begin{array}{ll}
x_{5} & x_{6} \\
x_{7} & x_{8}
\end{array}\right)
$$

satisfying relations

$$
\begin{gathered}
x_{1} x_{4}-x_{2} x_{3}-1=x_{5} x_{8}-x_{6} x_{7}-1=x_{2} x_{7}-x_{3} x_{6}=0 \\
-x_{2} x_{5}+x_{1} x_{6}-x_{4} x_{6}+x_{2} x_{8}=x_{3} x_{5}-x_{1} x_{7}+x_{4} x_{7}-x_{3} x_{8}=0 .
\end{gathered}
$$

The algebraic set $\operatorname{Hom}\left(\mathbb{Z}^{2}, S L(2, \mathbb{C})\right) \subset \mathbb{C}^{8}$ is irreducible by [Ri1, Thm. C]. 
For a more through study of representation varieties, it is useful to associate with each $\Gamma$ and $G$ as above an affine algebraic scheme, also called the representation variety, whose set of closed points coincides with $\operatorname{Hom}(\Gamma, G)$. That scheme, containing sometimes more subtle information about $G$-representations of $\Gamma$ than $\operatorname{Hom}(\Gamma, G)$, is constructed below.

If $G$ is an affine complex algebraic group, then $\mathbb{C}[G]$ is a Hopf algebra with the coproduct

$$
\Delta: \mathbb{C}[G] \rightarrow \mathbb{C}[G] \otimes \mathbb{C}[G]=\mathbb{C}[G \times G]
$$

being the dual to the group product $G \times G \rightarrow G$ and the antipode

$$
S: \mathbb{C}[G] \rightarrow \mathbb{C}[G]
$$

being the dual to the inverse map $g \rightarrow g^{-1}$. Consequently, for any commutative $\mathbb{C}$-algebra $A$ with product $m: A \times A \rightarrow A$, the space of algebra homomorphisms, $\operatorname{Hom}(\mathbb{C}[G], A)$, is a group with the multiplication

$$
\operatorname{Hom}(\mathbb{C}[G], A) \times \operatorname{Hom}(\mathbb{C}[G], A) \ni(f, g) \rightarrow m(f \otimes g) \Delta \in \operatorname{Hom}(\mathbb{C}[G], A)
$$

and the inverse

$$
\operatorname{Hom}(\mathbb{C}[G], A) \ni f \rightarrow f S \in \operatorname{Hom}(\mathbb{C}[G], A) .
$$

We will denote $\operatorname{Hom}(\mathbb{C}[G], A)$ with that group structure by $G(A)$. The functor $G(\cdot)$ is an affine group scheme, Wa. For example, $G(A)=S L(n, A)$ for $G=S L(n, \mathbb{C})$.

We say that a commutative $\mathbb{C}$-algebra $R(\Gamma, G)$ is a universal representation algebra of $\Gamma$ into $G$ and $\rho_{U}: \Gamma \rightarrow G(R(\Gamma, G))$ is a universal representation if for every commutative $\mathbb{C}$-algebra $A$ and every representation $\rho: \Gamma \rightarrow G(A)$, there is a $\mathbb{C}$-algebra homomorphism $f: R(\Gamma, G) \rightarrow A$ inducing a representation $G(f): G(R(\Gamma, G)) \rightarrow G(A)$ such that $\rho=G(f) \rho_{U}$, [BH, Si1].

Lemma 26. For every $\Gamma$ and every $G$ as above,

(1) $R(\Gamma, G)$ and $\rho_{U}$ exist.

(2) $R(\Gamma, G)$ is well defined up to an isomorphism of $\mathbb{C}$-algebras.

(3) $\rho_{U}: \Gamma \rightarrow G(R(\Gamma, G))$ is unique up to a composition with $G(f)$ where $f$ is a $\mathbb{C}$-algebra automorphism of $R(\Gamma, G)$.

Proof. (1) Since each affine algebraic group $G$ is a closed subgroup of $G L(n, \mathbb{C})$, the coordinate ring of $G$ is a quotient of

$$
\mathbb{C}[G L(n, \mathbb{C})]=\mathbb{C}\left[d, x_{i j}, 1 \leq i, j \leq n\right] /\left(d \cdot \operatorname{det}\left(x_{i j}\right)-1\right) .
$$

Let

$$
\mathbb{C}[G]=\mathbb{C}\left[d, x_{i j}, 1 \leq i, j \leq n\right] / I_{G}
$$

for an appropriate ideal $I_{G}$. For the free group, $F_{N}=\left\langle\gamma_{1}, \ldots, \gamma_{N}\right\rangle$,

$$
R\left(F_{N}, G\right)=\mathbb{C}\left[d_{1}, x_{1 i j}, 1 \leq i, j \leq n\right] / I_{G} \otimes \ldots \otimes \mathbb{C}\left[d_{N}, x_{N i j}, 1 \leq i, j \leq n\right] / I_{G}
$$

and

$$
\rho_{U}\left(\gamma_{t}\right)=\left(x_{t i j}\right) \in G(R(\Gamma, G)), \quad \text { for } t=1, \ldots, N
$$

satisfy the required universal properties.

If

$$
\Gamma=\left\langle\gamma_{1}, \ldots, \gamma_{N}\right\rangle / H
$$

then we define $R(\Gamma, G)$ as the quotient of $R\left(\left\langle\gamma_{1}, \ldots, \gamma_{N}\right\rangle, G\right)$ by an ideal $I$ generated by all relations necessary for (3) to be a well defined group homomorphism. Therefore, each normal generator of $H \triangleleft\left\langle\gamma_{1}, \ldots, \gamma_{N}\right\rangle$ introduces $n^{2}$ relations to $I$ 
(although some of them may be redundant). It is easy to see that, in this way, (3) descends to a universal representation $\rho_{U}: \Gamma \rightarrow G(R(\Gamma, G))$.

(2) and (3) follow immediately from the definition.

Every $\rho \in \operatorname{Hom}(\Gamma, G)$ defines a $\mathbb{C}$-algebra homomorphism $h_{\rho}: R(\Gamma, G) \rightarrow \mathbb{C}$ (unique up to an automorphism of $R(\Gamma, G)$ ) such that

$$
\rho=G\left(h_{\rho}\right) \rho_{U} .
$$

$\operatorname{Ker} h_{\rho}$ is a maximal ideal in $R(\Gamma, G)$ and, hence, a closed point in the affine scheme $\mathcal{H o m}(\Gamma, G)=\operatorname{Spec} R(\Gamma, G)$. Conversely, every closed point in $\mathcal{H o m}(\Gamma, G)$ defines a representation $\rho: \Gamma \rightarrow G$. Therefore, $\operatorname{Hom}(\Gamma, G)$ is the set of closed points of $\mathcal{H o m}(\Gamma, G)$ and there is a natural map

$$
\operatorname{Hom}(\Gamma, G) \rightarrow \mathcal{H o m}(\Gamma, G)
$$

dual to

$$
R(\Gamma, G) \rightarrow R(\Gamma, G) / \sqrt{0}=\mathbb{C}[\operatorname{Hom}(\Gamma, G)] .
$$

By [KM] Thm. 1.2], $R(\Gamma, P S L(2, \mathbb{C}))$ contains non-zero nilpotent elements for some Artin groups $\Gamma$. Furthermore, M. Kapovich proves that $R\left(\pi_{1}(M), S L(2, \mathbb{C})\right)$ and $R\left(\pi_{1}(M), P S L(2, \mathbb{C})\right)$ contain non-zero nilpotents for some 3-dimensional manifolds $M$, Ka1, Ka2]. See further comments in Section 12 ,

\section{SpaCES OF IRREDUCIBLE REPRESENTATIONS}

Proposition 27. For every $\Gamma$ and every reductive group $G$, the set of irreducible $G$-representations of $\Gamma$ is Zariski open in $\operatorname{Hom}(\Gamma, G)$.

Proof. The proposition follows from Corollary 17 and from [Ne, Prop. 3.8]. Since the proof of this referenced result is non-elementary, we enclose a complete simple proof here.

(1) First, a simple proof for $G=G L(n, \mathbb{C})$ and $S L(n, \mathbb{C})$ : If $\rho: \Gamma \rightarrow G$ is irreducible, then, by Shur's lemma, the elements of $\rho(\Gamma)$ linearly span the space of $n \times n$ matrices, $M(n, \mathbb{C})$. Conversely, if $\rho(\Gamma)$ lies in a parabolic subgroup of $G$, then the elements of $\rho(\Gamma)$ do not span $M(n, \mathbb{C})$.

Enumerate all elements of $\Gamma$ in a sequence $\gamma_{1}, \gamma_{2}, \ldots$. Let $U_{s}$ be the space of all $\rho$ 's such that $\rho\left(\gamma_{1}\right), \ldots, \rho\left(\gamma_{s}\right)$ span $M(n, \mathbb{C})$. Since the space of all irreducible $\rho$ 's is the union of all $U_{s}$ 's, it is enough to prove that each $U_{s}$ is open. This condition is equivalent to an existence of a sequence $i_{1}, \ldots, i_{n^{2}}$ such that the $n^{2} \times n^{2}$ matrix whose columns are $\rho\left(\gamma_{i_{1}}\right), \ldots, \rho\left(\gamma_{i_{n^{2}}}\right)$ considered as vectors in $M(n, \mathbb{C})=\mathbb{C}^{n^{2}}$ has a non-zero determinant. This is a Zariski open condition.

(2) Here is a fairly elementary proof for all $G$ :

The set of irreducible representations $\Gamma \rightarrow G$ is the complement of

$$
\bigcup_{P} \operatorname{Hom}(\Gamma, P) \subset \operatorname{Hom}(\Gamma, G),
$$

where the union on the left is over all proper parabolic subgroups of $G$. By [Bo, Thm. 14.18], there are only finitely many parabolic subgroups of $G$ up to conjugation. Therefore it is enough to prove that for a given $P$

$$
X_{P}=\bigcup_{g \in G} \operatorname{Hom}\left(\Gamma, g P g^{-1}\right) \subset \operatorname{Hom}(\Gamma, G)
$$


is closed. $X_{P}$ is the union of closed sets parameterized by a complete variety $G / P$. By the Projective Extension Theorem, [CLO, Ch. $8 \S 5$ Thm. 6], such a union is closed.

The adjoint representation induces a map $A d_{*}: \operatorname{Hom}(\Gamma, G) \rightarrow \operatorname{Hom}(\Gamma, G L(\mathfrak{g}))$. $\rho: \Gamma \rightarrow G$ is Ad-irreducible if $A d \rho$ is irreducible. Since the set of $A d$-irreducible representations $\Gamma \rightarrow G$ is the $A d_{*}$-preimage of the irreducible representations in $\operatorname{Hom}(\Gamma, G L(\mathfrak{g}))$, we conclude with

Corollary 28. The set of Ad-irreducible representations is Zariski open in $\operatorname{Hom}(\Gamma, G)$.

Proposition 29. Let $G$ be a reductive group.

(1) For a free group, $F_{N}$, of rank $N \geq 2$, the irreducible representations form a dense subset of $\operatorname{Hom}\left(F_{N}, G\right)$ (in complex topology).

(2) For every surface groun $\Gamma$, the irreducible representations are dense in a non-empty set of irreducible components of $\operatorname{Hom}(\Gamma, G)$.

Proof. (1) Since $\operatorname{Hom}\left(F_{N}, G\right)$ is an irreducible algebraic set and the set of irreducibles is Zariski open in it, it is enough to show that the set of irreducibles is non-empty. Since every free group $F_{N}$ of rank $N \geq 2$ maps onto $F_{2}$, it is enough to prove that statement for $F_{2}$. The set of irreducible $G$-representations of $F_{2}$ is the complement of

$$
\bigcup_{P} \operatorname{Hom}\left(F_{2}, P\right) \subset \operatorname{Hom}\left(F_{2}, G\right)=G \times G
$$

where the union of sets on the left is over all proper parabolic subgroups of $G$. By [Bo, Thm. 14.18], there are only finitely many parabolic subgroups of $G$ up to conjugation. Since for each $P$

$$
\bigcup_{g \in G} \operatorname{Hom}\left(F_{2}, g P g^{-1}\right)
$$

is the image of the $G$ action on $\operatorname{Hom}\left(F_{2}, P\right)$ with stabilizer $P$, its dimension is at most

$$
2 \cdot \operatorname{dim} P+\operatorname{dim} G-\operatorname{dim} P<2 \cdot \operatorname{dim} G=\operatorname{dim} \operatorname{Hom}\left(F_{2}, G\right) .
$$

Therefore, there exists an irreducible representation.

(2) Again, it is enough to prove that an irreducible representation exists. This follows from the fact that $\Gamma$ maps onto the free group of rank 2 .

Note that Proposition 29 does not apply to $F=$ torus. For example, all representations of $\mathbb{Z} \times \mathbb{Z}$ to $G L(n, \mathbb{C})$ are reducible for $n>1$.

\section{Stable and properly stable Representations in the sense of Git}

Let $O_{\rho}$ be the orbit of $\rho \in \operatorname{Hom}(\Gamma, G)$ under the $G$ action on $\operatorname{Hom}(\Gamma, G)$ by conjugation. In the language of geometric invariant theory, $\rho$ is poly-stable if $O_{\rho}$ is closed.

Theorem 30. For any reductive algebraic group $G, O_{\rho} \subset \operatorname{Hom}(\Gamma, G)$ is closed if and only if $\rho$ is completely reducible.

\footnotetext{
${ }^{1}$ A surface group is the fundamental group of a closed, orientable surface of genus $\geq 2$.
} 
Proof. (The proof for $G=G L(n, \mathbb{C})$ can be found in $[\mathrm{LM}$, Thm. 1.27]) $\Rightarrow$ We follow an argument of the proof of [JM, Thm. 1.1]: Assume that $O_{\rho}$ is closed. If $\rho$ is contained in a proper parabolic subgroup $P$, then by conjugating $\rho$ with a one parameter group in the center of a Levi subgroup $L$ of $P$ one can obtain a representation $\rho^{\prime} \in \bar{O}_{\rho}$ whose image lies in $L$. Since $O_{\rho}$ is closed, $\rho^{\prime}=g^{-1} \rho g$, for some $g \in P$. Hence $\rho$ lies in the Levi subgroup $g L g^{-1}$.

$\Leftarrow$ Any finitely generated group $\Gamma$ is a quotient of a free group $F$ of finite rank. Denote the epimorphism $F \rightarrow \Gamma$ by $\pi$. Since $\operatorname{Hom}(\Gamma, G)$ is a closed subset of $\operatorname{Hom}(F, G)$ and $O_{\rho}=O_{\pi \rho} \cap \operatorname{Hom}(\Gamma, G)$, it is enough to prove that $O_{\pi \rho} \subset \operatorname{Hom}(F, G)$ is closed. This statement follows from [Ri2, Thm. 3.6].

According to the geometric invariant theory, a point $x$ of an affine set $X$ is stable with respect to a $G$ action on $X$ (and the trivial line bundle on $X$ ) if there is a Zariski open neighborhood of $x$ preserved by $G$ on which the $G$ action is closed [MFK, Do].

Corollary 31. (1) Every irreducible representation is a stable point of $\operatorname{Hom}(\Gamma, G)$ under the $G$ action by conjugation.

(2) $\rho \in \operatorname{Hom}\left(F_{n}, G\right)$ is stable if and only if $\rho$ is irreducible.

Proof. (1) This follows from Proposition 27.

(2) Every stable $\rho$ is completely reducible by Theorem 30. Every completely reducible representation of a free group which is not irreducible can be deformed by an arbitrarily small deformation to a representation which is not completely reducible.

A point $x \in X$ is properly stable if it is stable and its stabilizer is finite.

Corollary 32. For every reductive group $G$,

(1) $\rho$ is a properly stable point of $\operatorname{Hom}(\Gamma, G)$ under conjugation action of $G$ if and only if $\rho$ is irreducible and $C(G)$ is finite.

(2) $\rho$ is a properly stable point of $H o m(\Gamma, G)$ under conjugation action of $G / C(G)$ if and only if $\rho$ is irreducible.

Proof. (1) $\Rightarrow: \rho$ is completely reducible by Theorem 30 , and it is irreducible by Lemma 16.

$\Leftarrow$ : By Theorem 30 and Propositions 27 and 15 ,

The same argument shows (2)

Following [JM], we say that a representation $\rho$ is good if $O_{\rho}$ is closed and $S_{\rho}$ is the center of $G$. By Theorem 30 and Corollary 17, every good representation is irreducible. By Proposition 14, every $A d$-irreducible representation is good.

Proposition 33. For every $\Gamma$ the space of good $G$-representations is open in the space of all $G$-representations of $\Gamma$.

Proof. By [JM, Proposition 1.1], the $G$ action on the space of all irreducible $G$ representations of $\Gamma$ is proper. The good representations, if they exist, form a set which is the the union of the principal orbits of that action. For every proper action, the union of principal orbits is an open subset, cf. [GO, Thm. 1.5]. 


\section{TANGENT SPACES}

Let $A$ be a commutative $\mathbb{C}$-algebra, let $m$ be a closed point of $S p e c A$, i.e., a maximal ideal $m \triangleleft A$, and let $r_{m}$ be the projection $A \rightarrow A / m=\mathbb{C}$. The tangent space to Spec $A$ at $m$ is the dual vector space to $m / m^{2}$,

$$
T_{m} \operatorname{Spec} A=\left(m / m^{2}\right)^{*} \text {. }
$$

Here is an equivalent definition of the tangent space which will be useful later: Let $\pi: \mathbb{C}[\varepsilon] /\left(\varepsilon^{2}\right) \rightarrow \mathbb{C}$ be the homomorphism sending $\varepsilon$ to 0 , and let $\mathcal{T}_{m}$ Spec $A$ be the complex vector space of $\mathbb{C}$-algebra homomorphisms $A \rightarrow \mathbb{C}[\varepsilon] /\left(\varepsilon^{2}\right)$ which descend to $r_{m}$ when composed with $\pi$. Since every such homomorphism is of the form $r_{m}+\tau \varepsilon$, where $\tau: A \rightarrow \mathbb{C}$ is a derivation,

$$
\mathcal{T}_{m} \operatorname{Spec} A=\left\{\tau: A \rightarrow \mathbb{C}: \tau(a b)=r_{m}(a) \tau(b)+r_{m}(b) \tau(a)\right\} .
$$

A straightforward calculation shows that for every $v \in T_{m} \operatorname{Spec} A$,

$$
\lambda_{v}(a)=v\left(a-r_{m}(a)\right)
$$

is a derivation in $\mathcal{T}_{m}$ SpecA. A direct computation shows that

$$
\lambda: T_{m} \operatorname{Spec} A \rightarrow \mathcal{T}_{m} \operatorname{Spec} A,
$$

sending $v$ to $\lambda_{v}$ is an isomorphism of vector spaces, [EH, VI.1.3]. From now on we will identify these two spaces and call them the Zariski tangent space to Spec A at $m$.

The above discussion applies to $A=\mathcal{H o m}(\Gamma, G)=\operatorname{Spec} R(\Gamma, G)$. Each $\rho \in$ $\operatorname{Hom}(\Gamma, G)$ defines a projection $r_{\rho}: R(\Gamma, G) \rightarrow \mathbb{C}$ and a closed point $m_{\rho}=\operatorname{Ker} \rho$ in $\mathcal{H o m}(\Gamma, G)$. We will abbreviate $T_{m_{\rho}} \mathcal{H o m}(\Gamma, G)$ to $T_{\rho} \mathcal{H o m}(\Gamma, G)$. Each tangent vector $\tau \in T_{\rho} \mathcal{H o m}(\Gamma, G)$ defines a group homomorphism

$$
\Gamma \stackrel{\rho_{U}}{\longrightarrow} G(R(\Gamma, G)) \stackrel{G\left(r_{\rho}+\tau \varepsilon\right)}{\longrightarrow} G\left(\mathbb{C}[\varepsilon] /\left(\varepsilon^{2}\right)\right) .
$$

By abuse of notation, we denote by $\pi$ the extension of the homomorphism

$$
\pi: \mathbb{C}[\varepsilon] /\left(\varepsilon^{2}\right) \rightarrow \mathbb{C}, \quad \pi(\varepsilon)=0,
$$

to the induced group homomorphism

$$
\pi: G\left(\mathbb{C}[\varepsilon] /\left(\varepsilon^{2}\right)\right) \rightarrow G(\mathbb{C})=G .
$$

Proposition 34. Consider a closed embedding $G \subset G L(n, \mathbb{C})$. (Such an embedding exists for every affine algebraic group.)

(1) For every $g \in G\left(\mathbb{C}[\varepsilon] /\left(\varepsilon^{2}\right)\right)$,

$$
\sigma(g)=\frac{g \cdot \pi(g)^{-1}-I}{\varepsilon} \in M_{n}\left(\mathbb{C}[\varepsilon] /\left(\varepsilon^{2}\right)\right),
$$

and $\sigma(g)$ belongs to the Lie algebra $\mathfrak{g} \subset M_{n}(\mathbb{C})$ of $G$.

(2) For every $g_{1}, g_{2} \in G\left(\mathbb{C}[\varepsilon] /\left(\varepsilon^{2}\right)\right)$,

$$
\sigma\left(g_{1} g_{2}\right)=\sigma\left(g_{1}\right)+A d \pi\left(g_{1}\right) \cdot \sigma\left(g_{2}\right),
$$

where $A d: G \rightarrow G L(\mathfrak{g})$ is the adjoint representation.

Proof. (1) If $h \in G\left(\mathbb{C}[\varepsilon] /\left(\varepsilon^{2}\right)\right)$ is such that $\pi(h)=I$, then $\frac{h-I}{\varepsilon}$ belongs to the Zariski tangent space to $G$ at the identity, that is, the Lie algebra of $G$. Now the statement follows by substitution $h=g \pi(g)^{-1}$.

(2) This follows by a direct computation. 
For every $\tau \in T_{\rho} \mathcal{H o m}(\Gamma, G), G\left(r_{\rho}+\tau \varepsilon\right) \rho_{U}(\gamma) \in G\left(\mathbb{C}[\varepsilon] /\left(\varepsilon^{2}\right)\right)$, cf. (6) . Therefore, by Proposition 34, we have a function $\sigma: \Gamma \rightarrow \mathfrak{g}$

$$
\sigma(\gamma)=\frac{\left(G\left(r_{\rho}+\tau \varepsilon\right) \rho_{U}(\gamma)\right)\left(G\left(r_{\rho}\right) \rho_{U}(\gamma)\right)^{-1}-I}{\varepsilon}
$$

satisfying (77), which is the cocycle condition for the first cohomology group of $\Gamma$ with coefficients in $\mathfrak{g}$ twisted by $A d \rho$. Hence, (8) defines a linear map

$$
\Psi_{\rho}: T_{\rho} \mathcal{H o m}(\Gamma, G) \rightarrow Z^{1}(\Gamma, A d \rho)
$$

sending $\tau$ to $\sigma$.

The adjoint action of the centralizer of $\rho(\Gamma), S_{\rho}$, on $\mathfrak{g}$ induces a $S_{\rho}$-action on $Z^{1}(\Gamma, A d \rho)$. Additionally, every $g \in S_{\rho}$ acts on $T_{\rho} \mathcal{H o m}(\Gamma, G)$ by sending $\tau \in$ $T_{\rho} \mathcal{H o m}(\Gamma, G)$ to $g \tau$ such that

$$
G\left(r_{\rho}+g \tau \varepsilon\right)=g G\left(r_{\rho}+\tau \varepsilon\right) g^{-1} .
$$

The homomorphism $\Psi_{\rho}$ is $S_{\rho}$-equivariant.

We are going to prove that $\Psi_{\rho}$ is an isomorphism by constructing its inverse. An easy calculation shows that for every $\sigma \in Z^{1}(\Gamma, A d \rho)$,

$$
\gamma \rightarrow(I+\sigma(\gamma) \varepsilon) \cdot \rho(\gamma)
$$

is a group homomorphism from $\Gamma$ to $G\left(\mathbb{C}[\varepsilon] /\left(\varepsilon^{2}\right)\right.$ ) (cf. [LM, Prop. 2.2] for $G=$ $G L(n, \mathbb{C}))$. Therefore, $\sigma$ defines a homomorphism $\Phi_{\rho}(\sigma): R(\Gamma, G) \rightarrow \mathbb{C}[\varepsilon] /\left(\varepsilon^{2}\right)$ such that $\pi \Phi_{\rho}(\sigma)=r_{\rho}$. Hence, $\Phi_{\rho}(\sigma) \in T_{\rho} \mathcal{H o m}(\Gamma, G)$. In other words, we have defined a linear map

$$
\Phi_{\rho}: Z^{1}(\Gamma, A d \rho) \rightarrow T_{\rho} \mathcal{H o m}(\Gamma, G)
$$

A straightforward computation shows (cf. [JM, Lem. 2.2], [LM, Prop. 2.2] for $G=G L(n, \mathbb{C})$ and $[$ Be, Prop. 1.2] for $G=P S L(2, \mathbb{C}))$ :

Theorem 35. $\Psi_{\rho}$ and $\Phi_{\rho}$ are inverses of each other, and therefore, they are $S_{\rho}$ equivariant isomorphisms between $Z^{1}(\Gamma, A d \rho)$ and $T_{\rho} \mathcal{H o m}(\Gamma, G)$.

\section{Smooth AND REDUCED REPRESENTATions}

A closed point $x$ of an algebraic scheme $X$ is reduced if the local ring $O_{X, x}$ has no non-zero nilpotent elements. By this definition, reduced points form a Zariski open subset of $X$.

Recall that a point $x$ of an affine algebraic set or of an algebraic scheme $X$ is simple if $x$ belongs to a unique irreducible component of $X$ and the dimension of that component coincides with $\operatorname{dim} T_{x} X$. Every simple point of an algebraic scheme is reduced.

We say that $\rho: \Gamma \rightarrow G$ is smooth (respectively, scheme smooth) if $\rho$ is a simple point of $\operatorname{Hom}(\Gamma, G)$ (respectively, of $\mathcal{H o m}(\Gamma, G)$ ). $\rho$ is reduced if $\rho$ is a reduced point of $\mathcal{H o m}(\Gamma, G)$. Note that $\rho$ is scheme smooth iff it is reduced and smooth.

Remark 36. (1) The set of all smooth representations,

(2) the set of all reduced representations,

(3) the set of all scheme smooth representations, are Zariski open subsets $\operatorname{Hom}(\Gamma, G)$. 
Proof. (1) This follows from the fact that the set of simple points are Zariski open.

(2) $\operatorname{Hom}^{r}(\Gamma, G)$ is a preimage of the Zariski open set of reduced points in $\mathcal{H o m}(\Gamma, G)$ under the map $\operatorname{Hom}(\Gamma, G) \rightarrow \mathcal{H o m}(\Gamma, G)$.

(3) This set is the intersection of the first two.

All $G$-representations of a free group are scheme smooth, since $R\left(F_{n}, G\right)$ is the coordinate ring of the $n$-th cartesian power of $G$, which is a non-singular algebraic set.

Proposition 37. For every reductive group $G$ and every surface group $\Gamma$, all irreducible representations $\rho: \Gamma \rightarrow G$ are scheme smooth and, hence, reduced.

Proof. By Proposition 15, the centralizer of $\rho(\Gamma)$ is a finite extension of the center of $G$. Hence, by Go1, Prop. 1.2] and Theorem 35.

$$
\operatorname{dim} T_{\rho} \mathcal{H o m}(\Gamma, G)=\operatorname{dim} Z^{1}(\Gamma, A d \rho)=(2 g-1) \operatorname{dim} G+\operatorname{dim} C(G) .
$$

(1) Assume first that $G$ is semi-simple. Then $\operatorname{dim} C(G)=0$. Since $\Gamma$ has a presentation with $2 g$ generators and one relation,

$$
\operatorname{dim} C \geq(2 g-1) \operatorname{dim} G
$$

for all irreducible components $C \subset \operatorname{Hom}(\Gamma, G)$. Therefore,

$$
\operatorname{dim} C \geq \operatorname{dim} T_{\rho} \mathcal{H o m}(\Gamma, G) .
$$

Since (10) must be an equality, $\rho$ is scheme smooth.

(2) For an arbitrary reductive group $G$, consider epimorphism (1),

$$
\nu: C^{0}(G) \times[G, G] \rightarrow G .
$$

Since it has a finite kernel, the induced map

$$
\operatorname{Hom}\left(\Gamma, C^{0}(G)\right) \times \operatorname{Hom}(\Gamma,[G, G]) \rightarrow \operatorname{Hom}(\Gamma, G)
$$

is finite. Since $\operatorname{Hom}\left(\Gamma, C^{0}(G)\right)=\left(C^{0}(G)\right)^{2 g}$ and, by $(1), \operatorname{Hom}(\Gamma,[G, G])$ is composed of irreducible components of dimension at least $(2 g-1) \operatorname{dim}[G, G]$, the set $\operatorname{Hom}(\Gamma, G)$ is composed of irreducible components of dimension at least

$$
2 g \cdot \operatorname{dim} C^{0}(G)+(2 g-1) \operatorname{dim}[G, G]=(2 g-1) \operatorname{dim} G+\operatorname{dim} C^{0}(G) .
$$

Therefore,

$$
\operatorname{dim} C \geq \operatorname{dim} T_{\rho} \mathcal{H o m}(\Gamma, G) .
$$

Now the argument goes exactly as in (1).

By [Shf, II $\S 2$ Thm. 6], we obtain:

Corollary 38. For every reductive group $G$, every irreducible representation of every surface group $\Gamma$ belongs to a unique irreducible component of $\operatorname{Hom}(\Gamma, G)$.

Proposition 39. If $G$ is a reductive group and $\Gamma$ is a surface group such that $\operatorname{Hom}(\Gamma, G)$ is irreducible, then $R(\Gamma, G)$ has no non-zero nilpotents. Consequently, map (4), $R(\Gamma, G) \rightarrow \mathbb{C}[\operatorname{Hom}(\Gamma, G)]$, is an isomorphism.

Proof. Recall from Section 8 that for every $\rho: \Gamma \rightarrow G$ there is a corresponding maximal ideal $m_{\rho} \triangleleft R(\Gamma, G)$. If we assume that $R(\Gamma, G)$ has a non-zero nilpotent, then that nilpotent projects onto a non-zero nilpotent in the localization $R(\Gamma, G)_{m_{\rho}}$ for an open set of $\rho$ 's in $\operatorname{Hom}(\Gamma, G)$. These $\rho$ 's are not reduced. 
Since $\operatorname{Hom}(\Gamma, G)$ is irreducible, by Proposition 29(2), the set of irreducible representations is dense in $\operatorname{Hom}(\Gamma, G)$. Therefore, $\Gamma$ has an irreducible but not reduced $G$-representation, contradicting Proposition 37

A version of this theorem for $F$ torus appears in [Si5].

Corollary 40. For $G=G L(n, \mathbb{C})$ and $S L(n, \mathbb{C})$ and every surface group $\Gamma$ the ring $R(\Gamma, G)$ is an integral domain, i.e., it has no proper zero divisors.

Proof. By Theorems 1 and 3 in $[\mathrm{RBC}, \operatorname{Hom}(\Gamma, G L(n, \mathbb{C}))$ and $\operatorname{Hom}(\Gamma, S L(n, \mathbb{C}))$ are irreducible. Therefore, the statement follows by Proposition 39

This corollary has some important consequences to the theory of skein modules, cf. Corollary 52, and it is needed to complete Goldman's construction of symplectic forms on character varieties of surfaces, cf. Section 14 .

The following remains open:

Question 41. Does $R(\Gamma, G)$ have non-zero nilpotents for some algebraic group $G$ and a surface group $\Gamma$ ?

Finally, we would like to remark that Proposition 37 does not hold for non-surface groups. In fact, there appears to be no easy characterization of simple points of $\operatorname{Hom}(\Gamma, G)$ in general.

Example 42. Let $\rho_{1}: \mathbb{Z}^{2} \rightarrow S L(2, \mathbb{C})$ be the trivial representation, and let $\rho_{2}$ : $\Gamma \rightarrow S L(2, \mathbb{C})$ be an irreducible representation. These representations define a representation $\rho_{1} * \rho_{2}: \mathbb{Z}^{2} * \Gamma \rightarrow S L(2, \mathbb{C})$ which is irreducible. On the other hand, the Jacobian matrix $\left(\partial r_{i} / \partial x_{j}\right)_{i=1, \ldots, 5, j=1, \ldots, 8}$ of the five relations in Example 25 has rank 2 at $\rho_{1}=\left(x_{1}, \ldots, x_{8}\right)=(1,0,0,1,1,0,0,1)$. Therefore, $\operatorname{Hom}\left(\mathbb{Z}^{2}, S L(2, \mathbb{C})\right)$ is singular at $\rho_{1}$ and, by Remark 24, $\rho_{1} * \rho_{2}$ is a singular point of $\operatorname{Hom}\left(\mathbb{Z}^{2} * \Gamma, G\right)$.

\section{ORBITS}

As before, let $O_{\rho}$ be the orbit of $\rho$ in $\operatorname{Hom}(\Gamma, G)$ under the $G$ action by conjugation. Since $O_{\rho}$ is homogeneous and (as every algebraic set) it has a simple point, all its points are simple, i.e., $O_{\rho}$ is a non-singular algebraic set.

The following theorem generalizes [JM, Lemma 2.2] and [LM, Cor. 2.4].

Theorem 43. For every $\rho$ the inclusion

$$
T_{\rho} O_{\rho} \subset T_{\rho} \mathcal{H o m}(\Gamma, G)
$$

corresponds to

$$
B^{1}(\Gamma, A d \rho) \subset Z^{1}(\Gamma, A d \rho)
$$

under the isomorphism $\Psi_{\rho}$.

Proof. Since $O_{\rho}$ is the image of the map $f_{\rho}: G \rightarrow \operatorname{Hom}(\Gamma, G), f_{\rho}(g)=g \rho g^{-1}, O_{\rho}$ is the left quotient of $G$ by the stabilizer of $\rho, S_{\rho}$, cf. [Bo, II.6.1]. Furthermore, the quotient map $G \rightarrow G / S_{\rho}$ induces an epimorphism $T_{[\rho]} G \rightarrow T_{\rho}\left(G / S_{\rho}\right)$. (Proof: $S_{\rho}$ acts on $G$ by left translation, with the trivial stabilizer. By Luna's Étale Slice Theorem, $\mathrm{Lu}, G \rightarrow G / / S_{\rho}$ is étale, inducing an isomorphism of tangent spaces. Note that $G / / S_{\rho}=G / S_{\rho}$ since all orbits are closed.) Therefore every tangent vector $v$ to $O_{\rho}$ at $\rho$ is of the form $d\left(f_{\rho}\right)(I+A \varepsilon)$. By the discussion of Section 8 , 
every vector $v \in T_{\rho} \mathcal{H o m}(\Gamma, G)$ corresponds to a group homomorphism (6). By this correspondence, $v$ corresponds to

$$
\gamma \rightarrow(I+A \varepsilon) \rho(\gamma)(I+A \varepsilon)^{-1} .
$$

Since

$$
(I+A \varepsilon)^{-1}=I-A \varepsilon \bmod \varepsilon^{2},
$$

(11) can be written as

$$
\gamma \rightarrow(I+A \varepsilon) \rho(\gamma)(I-A \varepsilon)=\left(I+\left(A-\rho(\gamma) A \rho(\gamma)^{-1}\right) \varepsilon\right) \rho(\gamma) \bmod \varepsilon^{2} .
$$

The right side is $(I+\tau \varepsilon) \rho(\gamma)$, where

$$
\tau=A-\rho(\gamma) A \rho(\gamma)^{-1} \in B^{1}(\Gamma, A d \rho) \subset Z^{1}(\Gamma, A d \rho) .
$$

Therefore, $\Psi_{\rho}$ of Theorem 35 maps $T_{\rho} O_{\rho}$ to $B^{1}(\Gamma, A d \rho)$. Since $\Psi_{\rho}$ is an embedding, in order to show $\Psi_{\rho}$ is an isomorphism from $T_{\rho} O_{\rho}$ to $B^{1}(\Gamma, A d \rho)$, it is enough to prove that

$$
\operatorname{dim} T_{\rho} O_{\rho} \geq \operatorname{dim} B^{1}(\Gamma, A d \rho) .
$$

Since $\operatorname{Ad} \rho(\gamma): \mathfrak{g} \rightarrow \mathfrak{g}$ is the differential of the map

$$
G \rightarrow G, \quad g \rightarrow \rho(\gamma) g \rho(\gamma)^{-1}
$$

and that map is constant on $S_{\rho}, A d \rho(\Gamma)$ acts trivially on the Lie algebra $L\left(S_{\rho}\right) \subset \mathfrak{g}$. Therefore (12) vanishes for $A \in L\left(S_{\rho}\right)$. Consequently,

$$
\operatorname{dim} B^{1}(\Gamma, A d \rho) \leq \operatorname{dim} \mathfrak{g}-\operatorname{dim} S_{\rho} .
$$

Since the left side of (13) is $\operatorname{dim} G-\operatorname{dim} S_{\rho}$, inequality (13) follows.

Remark 44. The action of $S_{\rho}$ on $T_{\rho} \mathcal{H} o m(\Gamma, G)$ and on $Z^{1}(\Gamma, A d \rho)$ preserves $T_{\rho} O_{\rho}$ and $B^{1}(\Gamma, A d \rho)$.

Theorems 35 and 43 imply Weil's infinitesimal rigidity theorem, cf. Wei].

Corollary 45. If $H^{1}(\Gamma, A d \rho)=0$, then

(1) $\rho: \Gamma \rightarrow G$ is scheme smooth (and, hence, reduced).

(2) $O_{\rho}$ contains an open neighborhood of $\rho$ (in complex topology) in $\operatorname{Hom}(\Gamma, G)$.

Proof. (1) Since $O_{\rho}$ is smooth, we have

$$
\begin{gathered}
\operatorname{dim} B^{1}(\Gamma, A d \rho)=\operatorname{dim} O_{\rho} \leq \operatorname{dim} \operatorname{Hom}(\Gamma, G) \leq \operatorname{dim} T_{\rho} H o m(\Gamma, G) \\
\leq \operatorname{dim} T_{\rho} \mathcal{H o m}(\Gamma, G)=\operatorname{dim} Z^{1}(\Gamma, \operatorname{Ad} \rho),
\end{gathered}
$$

by Theorems 35 and 43. Since all relations above are equalities, $\rho$ is scheme smooth.

(2) This follows from the fact that $\operatorname{Hom}(\Gamma, G)$ is smooth at $\rho$ and has the same dimension as $O_{\rho}$.

Since $H^{1}(\Gamma, A d \rho)=0$, for all finite groups $\Gamma$ and all $\rho$, cf. [Wb. Thm. 6.5.8], we also have:

Corollary 46. All G-representations of every finite group are reduced. 


\section{Character VARIEties}

The categorical quotient of $\operatorname{Hom}(\Gamma, G)$ by the $G$ action by conjugation,

$$
X_{G}(\Gamma)=\operatorname{Hom}(\Gamma, G) / / G,
$$

is called the $G$-character variety of $\Gamma$. By definition, it is an affine algebraic set together with the map $\operatorname{Hom}(\Gamma, G) \rightarrow \operatorname{Hom}(\Gamma, G) / / G$ which is constant on all $G$-orbits, with the property that every morphism from $\operatorname{Hom}(\Gamma, G)$ into an affine algebraic set $Y$ which is constant on all $G$-orbits factors through $\operatorname{Hom}(\Gamma, G) \rightarrow$ $\operatorname{Hom}(\Gamma, G) / / G$, cf. [Do, $\mathrm{Fo}, \mathrm{MFK}$. If $G$ is reductive, then the categorical quotient exists. The reason for considering the categorical quotient rather than the set theory quotient is that the quotient topology on $\operatorname{Hom}(\Gamma, G) / G$ is often not a Zariski topology of any algebraic set. In particular, it often contains points which are not closed. Character varieties are often reducible, despite being called "varieties".

Every equivalence class in $X_{G}(\Gamma)$ contains a unique closed orbit. Therefore, by Theorem 30, each element of the $G$-character variety of $\Gamma$ is represented by a unique completely reducible representation.

Example 47. Let $\mathbb{T}$ be a maximal torus of $G$. The map $\mathbb{T} \rightarrow H o m(\mathbb{Z}, G)$ sending $g$ to the $G$-representation $1 \rightarrow g$ of $\mathbb{Z}$ factors through an isomorphism

$$
\mathbb{T} / W \rightarrow X_{G}(\mathbb{Z}),
$$

where $W$ is the Weyl group of $G$, cf. [St, 6.4].

Example 48. (1) The $S L(2, \mathbb{C})$-character variety of the free group on two generators is isomorphic to $\mathbb{C}^{3}$.

(2) The $S L(3, \mathbb{C})$-character variety of the free group on two generators is a hypersurface in 9-dimensional affine space, [La1, Thm. 8], Si1].

Algebraic properties of character varieties are discussed in $\mathrm{AP}, \mathrm{BH}, \mathrm{BK} 1, \mathrm{BK} 2$, BK3, BKCh, GM, Ho1, Ho2, La1, La2, La3, La4, La5, LP, LM, Na, PBK, RBK, RBC, Si1, Si4, Si5, Wh]. Character varieties appear in many ways in mathematics and physics. Of particular importance are character varieties of surface groups, discussed in Section 14. They appear as moduli spaces of hyperbolic, projective, and other geometric structures on surfaces, cf. [BIW, Go1, Go3, Go9, GM1, GM2, GW, JM, KM, Li, Sa, Wa, as well as on the moduli spaces of flat connections, holomorphic bundles, and of Higgs bundles, AB, AMW, CHM, Da, DDW, DWWW, DWW, FL1, FL2, GGM, Hi1, Hi2, HLR, HT, Je2, Je3, JK, Ki, NS, Me, MW, O1, Rac, Sim1, Sim2, Th1, Th2, Th3, Wi2, Wi3, Za. These connections inspired an investigation of topology (and, more specifically, cohomology) of character varieties in many of the papers cited here.

In a related fashion, character varieties of surfaces also appear as examples of symplectic reduction (and, furthermore, Kähler and hyper-Kähler reductions) as well as in the context of Hamiltonian actions, $\mathrm{AB}, \mathrm{Au}, \mathrm{Go2}$, Je1. The action of the mapping class groups on character varieties is discussed in Go6, Go7, Go8, PX, SS, We, Wi.

In mathematical physics, character varieties appear in the context of Yang-Mills and Chern-Simons quantum field theories $\mathrm{At}, \mathrm{AB}, \mathrm{Ba}, \mathrm{Fr}, \mathrm{Gu}, \mathrm{JW}, \mathrm{KK}, \mathrm{We1}, \mathrm{We}$, We3, Wi1, Wi2, Wi3, as well as in the related skein theory of quantum invariants of 3-manifolds [Bu, CM, FG, FGL, Ga, Ge, Le2, PS1, PS2, Si2, Si3]. 
In low-dimensional topology, character varieties appear in the Bass-Culler-Shalen theory, in the context of $A$-polynomial and in other related areas $\mathrm{Be}, \mathrm{BB}, \mathrm{BLZ}$, BZ1, BZ2, BZ3, Du, DG, CCGLS, CL, CS, HS, MS, Mo, Shl] as well as in the context of Casson-Walker-Lescop invariant, $\mathrm{AM}, \mathrm{BC} 1, \mathrm{BC} 2, \mathrm{BHe}, \mathrm{BN}, \mathrm{Cu}$. Varieties of representations of 3-manifold groups have been studied for a long time also in relation to the Alexander polynomial, hyperbolic geometry, and for other independent reasons [BF, Ril1, HLM1, HLM2, HP1, HP2, Le1, LR1, LR2, Po, Ti]. Finally, character varieties appear in the discussion of local rigidity of discrete subgroups of Lie groups, cf. Corollary 45, Wei, Ra, Rag,.

These are just sample references to the above topics, as there are hundreds of papers devoted to every one of them.

Denote the set of irreducible representations in $\operatorname{Hom}(\Gamma, G)$ by $\operatorname{Hom}^{i}(\Gamma, G)$. The $G$ action by conjugation preserves this set. Since all orbits in $\operatorname{Hom}^{i}(\Gamma, G)$ are closed (cf. Theorem 30) and each equivalence class in a categorical quotient contains a unique closed orbit, the categorical quotient, $\operatorname{Hom}^{i}(\Gamma, G) / / G$, is the set-theoretic quotient as well. Denote it by $X_{G}^{i}(\Gamma)$.

Proposition 49. Let $G$ be a reductive group.

(1) For the free group $F_{n}, X_{G}^{i}\left(F_{n}\right)$ is a complex orbifold of complex dimension

$$
(n-1) \operatorname{dim} G+\operatorname{dim} C(G) \text {. }
$$

(2) For every closed orientable surface $S_{g}$ of genus $g>1, X_{G}^{i}\left(\pi_{1}\left(S_{g}\right)\right)$ is a complex orbifold of complex dimension

$$
(2 g-2) \operatorname{dim} G+2 \operatorname{dim} C(G) .
$$

(3) If $G$ is $C I$ (e.g., $G=G L(n, \mathbb{C}), S L(n, \mathbb{C}))$, then $X_{G}^{i}\left(F_{n}\right)$ and $X_{G}^{i}\left(\pi_{1}\left(S_{g}\right)\right)$ are manifolds for all $n, g>1$. (See also [FL2].)

Proof. (1) Since $\operatorname{Hom}^{i}\left(F_{n}, G\right)$ is an open subset of $G^{n}$, it is smooth. By JM, Prop. 1.1], the $G / C(G)$ action on $\operatorname{Hom}^{i}\left(F_{n}, G\right)$ is properly discontinuous. By Proposition 15. $G / C(G)$ acts on $\operatorname{Hom}^{i}\left(F_{n}, G\right)$ with finite centralizers. The quotient of a manifold by a properly discontinuous group action with finite stabilizers is an orbifold.

(2) By Proposition [37, all irreducible representations of $\pi_{1}\left(S_{g}\right)$ are scheme smooth. Therefore, by Theorem 35 and by [Go2, Prop. 1.2], $\operatorname{Hom}^{i}\left(\pi_{1}\left(S_{g}\right)\right)$ is a complex manifold of dimension $(2 g-1) \operatorname{dim} G+\operatorname{dim} C(G)$. Now the proof follows as in (1).

(3) By definition of a CI group, the $G / C(G)$ action on $X_{G}^{i}\left(F_{n}\right)$ is free. By [JM, Prop. 1.1], this action is also properly discontinuous. The quotient of a manifold by a free properly discontinuous group action is a manifold [Th, Proposition 3.5.7].

Recall that a representation $\rho: \Gamma \rightarrow G$ is good if and only if it is irreducible and the centralizer of $\rho(\Gamma)$ coincides with the center of $G$. By Proposition 33, the space of good representations, $\operatorname{Hom}^{g}(\Gamma, G)$, is an open subset of the irreducible ones. Since $G / C(G)$ acts freely and properly discontinuously on $\operatorname{Hom}^{g}(\Gamma, G)$, we have:

Corollary 50. For every reductive group $G$ and every surface group or a free group $\Gamma, X_{G}^{g}(\Gamma)=\operatorname{Hom}^{g}(\Gamma, G) / G$ is an open subset of $X_{G}^{i}(\Gamma)$ and a smooth complex manifold.

For a topological space $Y$, we will abbreviate $X_{G}\left(\pi_{1}(Y)\right)$ by $X_{G}(Y)$. 
Proposition 51. (1) If $M$ is a compact 3-manifold with a connected boundary of genus $g$, then

$$
\operatorname{dim} X_{G}(M) \geq \operatorname{dim} G \cdot(g-1)+\operatorname{dim} C(G) .
$$

(2) For a given non-abelian reductive group $G$ and a positive integer $g$, there is no upper bound on $\operatorname{dim} X_{G}(M)$ over compact 3-manifolds $M$ with connected boundary of fixed genus $g$.

Proof. (1) If $M$ is a compact manifold with connected boundary of genus $g$, then $\pi_{1}(M)$ has a presentation with $n$ generators and $p$ relations such that

$$
1-n+p=\chi(M)=1-g .
$$

Hence $\operatorname{dim} \operatorname{Hom}\left(\pi_{1}(M), G\right) \geq \operatorname{dim} G \cdot(n-p)=\operatorname{dim} G \cdot g$. Since $X_{G}(M)$ is the quotient of $\operatorname{Hom}\left(\pi_{1}(M), G\right)$ by $G / C(G)$, the statement follows.

(2) It is enough to prove that there is no upper bound on $\operatorname{dim} \operatorname{Hom}\left(\pi_{1}(M), G\right)$ over compact manifolds $M$ with connected boundary of fixed genus $g$. Since every non-abelian reductive group contains either $S L(2, \mathbb{C})$ or $P S L(2, \mathbb{C})$,

$$
\begin{aligned}
\operatorname{dim} \operatorname{Hom} & \left(\pi_{1}(M), G\right) \\
& \geq \min \left(\operatorname{dim} \operatorname{Hom}\left(\pi_{1}(M), S L(2, \mathbb{C})\right), \operatorname{dim} \operatorname{Hom}\left(\pi_{1}(M), \operatorname{PSL}(2, \mathbb{C})\right) .\right.
\end{aligned}
$$

Since the quotient map $S L(2, \mathbb{C}) \rightarrow P S L(2, \mathbb{C})$ induces a finite map

$$
\begin{aligned}
& \operatorname{Hom}\left(\pi_{1}(M), S L(2, \mathbb{C})\right) \rightarrow \operatorname{Hom}\left(\pi_{1}(M), P S L(2, \mathbb{C})\right), \\
& \operatorname{dim} \operatorname{Hom}\left(\pi_{1}(M), S L(2, \mathbb{C})\right) \leq \operatorname{dim} \operatorname{Hom}\left(\pi_{1}(M), P S L(2, \mathbb{C})\right) .
\end{aligned}
$$

(The inequality stems from the fact that this map does not have to be onto.) Therefore, it is enough to prove that there is no upper bound on

$$
\operatorname{dim} \operatorname{Hom}\left(\pi_{1}(M), S L(2, \mathbb{C})\right) \text {. }
$$

Let $K_{n}$ be the connected sum of $n$ copies of a knot $K$. Cooper and Long [CL] proved that $\operatorname{dim} \operatorname{Hom}\left(\pi_{1}\left(S^{3} \backslash K_{n}\right), S L(2, \mathbb{C})\right) \geq n+3$. (Although their argument is made for hyperbolic knots $K$ only, it generalizes to all knots by the result of [KrM], cf. [DG.) Let $K_{n, g}$ be a graph obtained by connecting $g$ unlinked copies of $K_{n}$ in $S^{3}$ by $g-1$ tunnels, and let $M_{n, g}$ be the complement of an open tabular neighborhood of $K_{n, g}$ in $S^{3}$. Then $\pi_{1}\left(M_{n, g}\right)$ is the free product of $g$ copies of $\pi_{1}\left(S^{3} \backslash K_{n}\right)$ and

$$
\operatorname{dim} \operatorname{Hom}\left(\pi_{1}\left(M_{n, g}\right), S L(2, \mathbb{C})\right) \geq g \cdot(n+3),
$$

by Remark 24. Since $\partial M_{n, g}$ is a surface of genus $g$, the statement follows.

\section{Character varieties as algebraic schemes}

The algebraic scheme $\mathcal{X}_{G}(\Gamma)=\mathcal{H o m}(\Gamma, G) / / G$ is often also called the $G$-character variety of $\Gamma$. By the definition of categorical quotient, $\mathcal{X}_{G}(\Gamma)=\operatorname{Spec} R(\Gamma, G)^{G}$. The epimorphism $R(\Gamma, G) \rightarrow \mathbb{C}[\operatorname{Hom}(\Gamma, G)]$ induces the map

$$
R(\Gamma, G)^{G} \rightarrow \mathbb{C}[\operatorname{Hom}(\Gamma, G)]^{G},
$$

which is an epimorphism by the properties of the Reynolds operator. Hence, we have an embedding of schemes

$$
i: X_{G}(\Gamma) \hookrightarrow \mathcal{X}_{G}(\Gamma) .
$$

Since the kernel of (14) is the nil-radical of $R(\Gamma, G)^{G}$, (15) is a bijection between the points of $X_{G}(\Gamma)$ and the closed points of $\mathcal{X}_{G}(\Gamma)$. 
In [Si1], we have described the coordinate ring of $\mathcal{X}_{S L(n, \mathbb{C})}(\Gamma)$ as a space of $n$ valent graphs reminiscent of Feynman diagrams in an arbitrary path connected topological space $X$ with $\pi_{1}(X)=\Gamma$.

By Corollary 40, $R(\Gamma, G)^{G}$ has no zero divisors for surface groups and $G=$ $G L(n, \mathbb{C}), S L(n, \mathbb{C})$. Hence, by $\mathrm{RBC}, \mathcal{X}_{G}(\Gamma)$ is reduced and irreducible for such groups. For the $F$ torus and $G=S L(2, \mathbb{C}), R\left(\pi_{1}(F), G\right)^{G}$ is reduced as well by [PS1, Thm. 3.3]. These facts have an important consequence for the theory of skein modules.

Corollary 52. For every closed orientable surface $F$, the map $\phi$ of PS2, Thm. 7.1] is an isomorphism between the skein algebra of $\pi_{1}(F)$ and $\mathbb{C}\left[X_{S L(2, \mathbb{C})}\left(\pi_{1}(F)\right)\right]$. Consequently, $\phi$ composed with the isomorphism of [PS2, Thm. 2.8] is an isomorphism from the skein algebra of a surface, $S_{2, \infty}(F, \mathbb{C},-1)$, to $\mathbb{C}\left[X_{S L(2, \mathbb{C})}\left(\pi_{1}(F)\right)\right]$.

Although this result was announced in [PS2, Thm. 7.3], its proof required [PS2, Thm. 4.7] whose proof was not provided. An alternative proof of the above statement was provided independently by L. Charles and J. Marché in [CM].

$\mathcal{X}_{G}(\Gamma)$ is not always reduced. Kapovich and Millson proved that for every affine (possibly unreduced) variety $X$ over $\mathbb{Q}$ there is an Artin group $\Gamma$ such that a Zariski open subset of $\mathcal{X}_{P S L(2, \mathbb{C})}(\Gamma)$ is isomorphic to a Zariski open subset of $X[\mathrm{KM}$. Additionally, for every $x \in X$ there is a representation $\rho$ of an Artin group $\Gamma$ into $\operatorname{PSL}(2, \mathbb{C})$ such that the analytic germ of $\mathcal{X}_{P S L(2, \mathbb{C})}(\Gamma)$ at $[\rho]$ coincides with the analytic germ of $X$ at $x[\mathrm{KM}]$.

Kapovich proved that the same is true for 3-manifold groups. That is, for every $x \in X$ as above there is a closed 3 -manifold $M$ and a $P S L(2, \mathbb{C})$-representation $\rho$ of $\Gamma=\pi_{1}(M)$ such that the analytic germ of $\mathcal{X}_{P S L(2, \mathbb{C})}(\Gamma)$ at $[\rho]$ coincides with the analytic germ of $X$ at $x$ Ka1, Ka2. In particular $\mathcal{X}_{P S L(2, \mathbb{C})}(\Gamma)$ contains non-zero nilpotent elements for some Artin groups and some 3-manifold groups $\Gamma$.

\section{TANGEnT SPACES TO CHARACTER VARIETIES}

For every $\rho$ the map (15) induces an embedding

$$
T_{[\rho]} X_{G}(\Gamma) \hookrightarrow T_{[\rho]} \mathcal{X}_{G}(\Gamma) .
$$

It is an isomorphism if $\rho$ is reduced. We are going to give a cohomological description of these tangent spaces.

For every $\rho: \Gamma \rightarrow G$, the $S_{\rho}$ action on $Z^{1}(\Gamma, A d \rho)$ descends to an action on $H^{1}(\Gamma, A d \rho)$.

Theorem 53. (1) If $\rho: \Gamma \rightarrow G$ is completely reducible, then the isomorphism $\Phi_{\rho}$ of Theorem 35 combined with the natural projection $\mathcal{H} o m(\Gamma, G) \rightarrow \mathcal{X}_{G}(\Gamma)$ induces a natural linear map

$$
\phi: H^{1}(\Gamma, A d \rho) \rightarrow T_{[\rho]} \mathcal{X}_{G}(\Gamma) .
$$

(2) If $\rho$ is good, then $\phi$ is an isomorphism.

(3) If $\rho$ is completely reducible and scheme smooth (but not necessarily good), then

$$
\operatorname{dim} T_{0}\left(H^{1}(\Gamma, A d \rho) / / S_{\rho}\right)=\operatorname{dim} T_{[\rho]} \mathcal{X}_{G}(\Gamma)=\operatorname{dim} T_{[\rho]} X_{G}(\Gamma) .
$$

The naturality of the morphism $\phi$ means that for every $\alpha: \Gamma^{\prime} \rightarrow \Gamma$ and $\beta: G \rightarrow$ $G^{\prime}$ such that $\rho: \Gamma \rightarrow G$ and $\beta \rho \alpha: \Gamma^{\prime} \rightarrow G^{\prime}$ are completely reducible, the following 
diagram commutes:

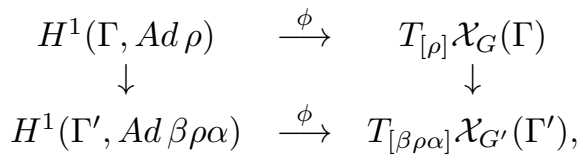

where the vertical homomorphisms are induced by $\alpha$ and $\beta$.

We discuss the existence of a natural isomorphism in part (3) of the above theorem at the end of this section.

Versions of the above theorem for $G=P S L(2, \mathbb{C})$ appear in Po, Prop. 3.5] and [HP2, Prop. 5.2]. (Note that every irreducible $P S L(2, \mathbb{C})$-representation is $A d$ irreducible and, hence, good, by Proposition 14.) A related discussion (for abelian representations) appears in $\mathrm{Be}$.

Although statements similar to Theorem 53(2) appear in the literature for general $G$, there is a lot of confusion about the necessary assumptions and there is a lack of discussion of the naturality of the isomorphism. Furthermore, all proofs known to us are incomplete.

Proof of Theorem [53. The proof of the above theorem is based on Luna's Étale Slice Theorem, $[\mathrm{Lu}$, cf. [MFK, [PV, Thm. 6.1], which applies to closed orbits of group actions on affine (not necessarily reduced) schemes.

(1) Since the orbit of $\rho$ is closed by Theorem 30, by Luna's Slice Theorem there exists a subscheme $S \subset \mathcal{H o m}(\Gamma, G)$, called an étale slice, such that

(i) $\rho \in S$.

(ii) $S$ is preserved by the $S_{\rho}$ action.

(iii) If we denote the categorical quotient of $G \times S$ by the diagonal $S_{\rho}$-action (which is right on $G$ and left on $S$ ) by $G \times \times_{S_{\rho}} S$, then the natural map $G \times{ }_{S_{\rho}} S \rightarrow \mathcal{H o m}(\Gamma, G)$ sending $(g, s)$ to $g s$ is étale.

(iv) $S / / S_{\rho} \rightarrow \mathcal{X}_{G}(\Gamma)$ is étale.

In particular, it means that the differentials of the above maps

$$
T_{(e, \rho)}\left(G \times_{S_{\rho}} S\right) \rightarrow T_{\rho} \mathcal{H o m}(\Gamma, G)
$$

and

$$
T_{[\rho]} S / / S_{\rho} \rightarrow T_{[\rho]} \mathcal{X}_{G}(\Gamma)
$$

are isomorphisms.

The natural projection,

$$
\mathcal{H o m}(\Gamma, G) \rightarrow \mathcal{X}_{G}(\Gamma)
$$

induces

$$
T_{\rho} \mathcal{H o m}(\Gamma, G) \rightarrow T_{[\rho]} \mathcal{X}_{G}(\Gamma),
$$

which can be written as

$$
T_{\rho} \mathcal{H o m}(\Gamma, G) \stackrel{\simeq}{\longrightarrow} T_{(e, \rho)}\left(G \times_{S_{\rho}} S\right) \rightarrow T_{[\rho]}\left(S / / S_{\rho}\right) \stackrel{\simeq}{\longrightarrow} T_{[\rho]} \mathcal{X}_{G}(\Gamma),
$$

where the first map is the inverse of (17), the second is the differential of

$$
G \times{ }_{S_{\rho}} S \rightarrow S / / S_{\rho}
$$

and the third one is (18). 
Since the map $G \rightarrow G \times \times_{S_{\rho}} S$ composed with (21) is constant, the image of $T_{e} G$ in $T_{(e, \rho)} G \times_{S_{\rho}} S$ belongs to the kernel of $T_{\rho} \mathcal{H o m}(\Gamma, G) \rightarrow T_{\rho}\left(S / / S_{\rho}\right)$. Since this image corresponds to $T_{\rho} O_{\rho} \subset T_{\rho} \mathcal{H o m}(\Gamma, G)$, (20) factors through

$$
T_{\rho} \mathcal{H o m}(\Gamma, G) / T_{\rho} O_{\rho} \rightarrow T_{\rho}\left(S / / S_{\rho}\right) \stackrel{\simeq}{\longrightarrow} T_{\rho} \mathcal{X}_{G}(\Gamma) .
$$

Now the statement follows from Theorems 35 and 43 ,

(2) If $\rho$ is good, then $S_{\rho}=C(G)$ acts trivially on $S$ and (21) can be written as the projection

$$
G / C(G) \times S \rightarrow S
$$

Its differential is the projection $T_{e} G / C(G) \times T_{\rho} S \rightarrow T_{\rho} S$ whose kernel is $T_{\rho} O_{\rho}$. Therefore, the left morphism of (22) is an isomorphism.

(3) Since the stabilizer of the $S_{\rho}$-action on $G \times S$ at $(e, \rho)$ is trivial, by Luna's Slice Theorem we have an étale morphism $(G \times S) /\{e\} \rightarrow G \times S_{\rho} S$ yielding an isomorphism of the tangent spaces. Composing it with (17), we obtain an isomorphism

$$
T_{e} G \times T_{\rho} S \rightarrow T_{\rho} \mathcal{H o m}(\Gamma, G) .
$$

The image of $T_{e} G$ in $T_{\rho} \mathcal{H o m}(\Gamma, G)$ is $T_{\rho} O_{\rho}$. Therefore,

$$
T_{\rho} S \simeq \mathcal{H o m}(\Gamma, G) / T_{\rho} O_{\rho} \simeq H^{1}(\Gamma, A d \rho) .
$$

Since $\rho$ is a simple point of $\mathcal{H o m}(\Gamma, G)$, we can assume that $S$ is non-singular at $\rho$ by [Lu, Remark III.1.1]. Therefore, there exists an isomorphism

$$
T_{\rho}\left(S / / S_{\rho}\right) \simeq T_{0}\left(T_{\rho} S / / S_{\rho}\right)
$$

by [Lu, Lemma III.1] (cf. [PV, Thm. 6.4]). This isomorphism combined with (24) and (18) implies the statement.

Since $O_{\rho}$ is always reduced, it is a subvariety of $\operatorname{Hom}(\Gamma, G)$ in $\mathcal{H o m}(\Gamma, G)$. A version of the above argument applied to the $G$ action on $\operatorname{Hom}(\Gamma, G)$ yields the following:

Theorem 54. (1) If $\rho$ is completely reducible, then the projection $\operatorname{Hom}(\Gamma, G) \rightarrow$ $X_{G}(\Gamma)$ induces a linear map

$$
T_{\rho} \operatorname{Hom}(\Gamma, G) / B^{1}(\Gamma, A d \rho) \rightarrow T_{[\rho]} X_{G}(\Gamma) .
$$

(2) If $\rho$ is good, then this is an isomorphism.

(3) If $\rho$ is smooth, then

$$
T_{0}\left(\left(T_{\rho} \operatorname{Hom}(\Gamma, G) / B^{1}(\Gamma, A d \rho)\right) / / S_{\rho}\right) \simeq T_{[\rho]} X_{G}(\Gamma) .
$$

Corollary 55. If $\rho$ is good, then $\mathcal{X}_{G}(\Gamma)$ is reduced at $[\rho]$ if and only if $\rho$ is reduced.

Proof. $\mathcal{X}_{G}(\Gamma)$ is reduced if and only if

$$
T_{[\rho]} X_{G}(\Gamma)=T_{[\rho]} \mathcal{X}_{G}(\Gamma)
$$

which holds if and only if

$$
T_{\rho} \operatorname{Hom}(\Gamma, G)=T_{\rho} \mathcal{H o m}(\Gamma, G)
$$

by Theorems 553(2) and 54(2). 
Question 56. (1) Is there a natural linear map

$$
T_{0}\left(H^{1}(\Gamma, A d \rho) / / S_{\rho}\right) \rightarrow T_{[\rho]} \mathcal{X}_{G}(\Gamma)
$$

for all completely reducible $\rho$, which is an isomorphism for scheme smooth $\rho$ ?

(2) Is this map an injection or surjection for $\rho$ which are not scheme smooth?

The difficulty in answering this question comes from the following three factors:

(1) Étale slices are not unique.

(2) The isomorphism (25) is non-canonical for a given slice $S$.

(3) Map $\phi$ does not descend to an isomorphism

$$
\left.T_{0}\left(H^{1}(\Gamma, A d \rho) / / S_{\rho}\right)\right) \rightarrow T_{[\rho]} \mathcal{X}_{G}(\Gamma)
$$

if $H^{1}(\Gamma, A d \rho) \rightarrow T_{0}\left(H^{1}(\Gamma, A d \rho) / / S_{\rho}\right)$ is not onto.

The following result illuminates the importance of the requirement of $\rho$ being scheme smooth in Theorem 53(2).

Theorem 57. $\mathcal{X}_{G}^{g}(\Gamma)$ is reduced iff $T_{[\rho]} X_{G}(\Gamma)=H^{1}(\Gamma, A d \rho)$ for a non-empty Zariski open set of $[\rho]$ 's in $X_{G}^{g}(\Gamma)$.

Proof. $\Rightarrow$ By Theorem $53(2)$, the equality holds for all $[\rho] \in X_{G}^{g}(\Gamma)$.

$\Leftarrow$ If $\mathcal{X}_{G}^{g}(\Gamma)$ is not reduced, then $\mathcal{X}_{G}^{g}(\Gamma)$ is not reduced at $[\rho]$ for an open set $\Omega$ of $[\rho]$ 's in $X_{G}^{g}(\Gamma)$. Since the set of smooth and good representations, $\operatorname{Hom}^{s g}(\Gamma, G)$, is the non-singular part of the set of good representations, it is an open dense subset of $\operatorname{Hom}^{g}(\Gamma, G)$. Hence $\Omega \cap \operatorname{Hom}^{s g}(\Gamma, G)$ is non-empty and open.

By Corollary [55, $\rho$ 's in $\Omega \cap H_{o m}^{s g}(\Gamma, G)$ are not reduced. Therefore,

$$
\operatorname{dim} T_{\rho} \operatorname{Hom}(\Gamma, G)<\operatorname{dim} T_{\rho} \mathcal{H o m}(\Gamma, G)=\operatorname{dim} Z^{1}(\Gamma, A d \rho)
$$

by Theorem 35. Since

$$
\operatorname{dim} T_{[\rho]} X_{G}(\Gamma)=\operatorname{dim} T_{\rho} \operatorname{Hom}(\Gamma, G)-\operatorname{dim} B^{1}(\Gamma, A d \rho)
$$

for $\rho \in \operatorname{Hom}^{s g}(\Gamma, G)$, by Theorem 54 (3), we get

$$
\operatorname{dim} T_{[\rho]} X_{G}(\Gamma)<\operatorname{dim} H^{1}(\Gamma, A d \rho)
$$

for $\rho \in \Omega \cap H o m{ }^{s g}(\Gamma, G)$.

\section{Symplecticity of Character varieties of SURFACES}

Let $G$ be a reductive group, and let $\mathfrak{g}$ be its Lie algebra. A bilinear form $B: \mathfrak{g} \times \mathfrak{g} \rightarrow \mathbb{C}$ is $A d$-invariant if $B(A d(g) x, A d(g) y)=B(x, y)$.

Let $F$ be a closed orientable surface. For every representation $\rho: \pi_{1}(F) \rightarrow G$ and every $A d$-invariant bilinear form $B: \mathfrak{g} \times \mathfrak{g} \rightarrow \mathbb{C}$, the cup product defines a pairing

(26) $\omega_{B}: H^{1}(F, A d \rho) \times H^{1}(F, A d \rho) \stackrel{\cup}{\longrightarrow} H^{2}(F, A d \rho \otimes A d \rho) \stackrel{B}{\longrightarrow} H^{2}(F, \mathbb{C})=\mathbb{C}$, which can also be identified with the pairing

$$
H^{1}(F, A d \rho) \times H_{1}(F, A d \rho) \stackrel{\cap}{\longrightarrow} H_{0}(F, A d \rho \otimes A d \rho) \stackrel{B}{\longrightarrow} H_{0}(F, \mathbb{C})=\mathbb{C}
$$

via the Poincaré duality with twisted coefficients, $\mathrm{Sp}$,

$$
\cap[F]: H^{n}(F, A d \rho) \rightarrow H_{2-n}(F, A d \rho),
$$

where $[F] \in H_{2}(F, \mathbb{C})$ is a fundamental class of $F$. 
Let $\Gamma$ be a group, and let $\left(C_{*}, \partial\right)$ be a chain complex of left $\mathbb{Z} \Gamma$-modules. Let $M_{1}, M_{2}$ be left $\mathbb{Z} \Gamma$-modules. If $B: M_{1} \times M_{2} \rightarrow \mathbb{C}$ is a $\mathbb{Z} \Gamma$-invariant pairing, i.e., $B\left(r m_{1}, r m_{2}\right)=B\left(m_{1}, m_{2}\right)$ for every $r \in \mathbb{Z} \Gamma, m_{1} \in M_{1}, m_{2} \in M_{2}$, then, by [Br, $\mathrm{V}$ $\S 3]$, the cap product induces a pairing

$$
H^{n}\left(H_{\mathrm{oom}}\left(C_{*}, M_{2}\right), \delta\right) \times H_{n}\left(M_{1} \otimes_{\mathbb{Z} \Gamma} C_{*}, \partial\right) \rightarrow M_{1} \otimes_{\mathbb{Z} \Gamma} M_{2} \stackrel{B}{\longrightarrow} \mathbb{C} .
$$

In the above formula $M_{1}$ is considered as a right $\mathbb{Z} \Gamma$-module via $m \cdot \gamma=\gamma^{-1} \cdot m$.

Lemma 58. If $B$ is a duality pairing, i.e., if $B$ induces an isomorphism $M_{1} \simeq$ $\operatorname{Hom}\left(M_{2}, \mathbb{C}\right)$, then the pairing (28) is non-degenerate.

Proof. The cochain complex $\left(H_{\mathrm{om}}\left(\mathrm{C}_{\mathbb{Z}}, M_{2}\right), \delta\right)$ can be written as

$$
\begin{gathered}
\left(\operatorname{Hom}_{\mathbb{Z} \Gamma}\left(C_{*}, \operatorname{Hom}\left(M_{1}, \mathbb{C}\right)\right), \delta\right)=\left(\operatorname{Hom}_{\mathbb{Z} \Gamma}\left(C_{*} \otimes M_{1}, \mathbb{C}\right), \delta\right) \\
=\left(\operatorname{Hom}\left(C_{*} \otimes_{\mathbb{Z} \Gamma} M_{1}, \mathbb{C}\right), \delta\right)=\operatorname{Hom}\left(\left(C_{*} \otimes_{\mathbb{Z} \Gamma} M_{1}, \partial\right), \mathbb{C}\right) .
\end{gathered}
$$

Hence

$$
H_{n}\left(\operatorname{Hom}_{\mathbb{Z} \Gamma}\left(C_{*}, M_{2}\right), \delta\right)=H_{n}\left(H o m\left(\left(C_{*} \otimes_{\mathbb{Z} \Gamma} M_{1}, \partial\right), \mathbb{C}\right)\right) .
$$

Since $\mathbb{C}$ is a divisible group, $\operatorname{Hom}(\cdot, \mathbb{C})$ is an exact functor in the category of abelian groups. Hence, (29) becomes

$$
H_{n}\left(\operatorname{Hom}_{\mathbb{Z} \Gamma}\left(C_{*}, M_{2}\right), \delta\right)=\operatorname{Hom}\left(H_{n}\left(C_{*} \otimes_{\mathbb{Z} \Gamma} M_{1}, \partial\right), \mathbb{C}\right) .
$$

It is easy to verify that this isomorphism is induced by (28).

If $B$ is symmetric, then (26) is skew-symmetric. Therefore, Lemma 58 implies:

Corollary 59 (cf. Go1]). If $B: \mathfrak{g} \times \mathfrak{g} \rightarrow \mathbb{C}$ is symmetric, Ad-invariant, and non-degenerate, then (26) is a symplectic form on $H^{1}(F, A d \rho)$.

If $\mathfrak{g}$ is simple, then the Killing form is unique among symmetric, $G$-invariant, non-degenerate forms on $\mathfrak{g}$, up to a constant multiple.

Let $F$ be a closed orientable surface of genus $\geq 2$ now. By Corollary 50 , the space of conjugacy classes of good representations,

$$
X_{G}^{g}(F)=H_{o m}^{g}\left(\pi_{1}(F), G\right) / / G=H_{o m}^{g}\left(\pi_{1}(F), G\right) / G
$$

is a complex manifold and, by Proposition 37 and Theorem 53(2),

$$
T_{[\rho]} X_{G}^{g}(F)=H^{1}\left(\pi_{1}(F), A d \rho\right) .
$$

Remark 60. Goldman gave an "algebraic" description of the form $\omega_{B}$ on $X_{G}^{g}(F)$, Go2 . His argument implies that $\omega_{B}$ is holomorphic. However, we do not know if $\omega_{B}$ can be written as $\sum_{i=1}^{n} f_{i} d g_{i} \wedge d h_{i}$, for some regular functions $f_{1}, \ldots, f_{n}$, $g_{1}, \ldots, g_{n}, h_{1}, \ldots, h_{n}$ on $X_{G}(F)$.

Goldman proves by an argument from gauge theory that for every non-degenerate, symmetric, $A d$-invariant $B, \omega_{B}$ is closed [Go1]. Therefore, $\left(X_{G}^{g}(F), \omega_{B}\right)$ is a holomorphic symplectic manifold. (Note that our Proposition 37 is needed to complete Goldman's construction of $\omega_{B}$.)

Although $X_{G}^{g}$ (torus) is empty for most $G, X_{G}$ (torus) is a singular symplectic manifold as well [Si5]. 


\section{3-MANIFOLDS AND LAGRANGIAN SUBSPACES}

Let $M$ be an orientable compact 3-manifold with a connected boundary $F$. The embedding $\partial M \hookrightarrow M$ induces a homomorphism $r: \pi_{1}(F) \rightarrow \pi_{1}(M)$ and a map $r^{*}: X_{G}(M) \rightarrow X_{G}(F)$.

It is often believed that the non-singular part of the image of $X_{G}(M)$ in $X_{G}^{g}(F)$ is Lagrangian. We investigate this claim in this section.

Theorem 61. Consider the symplectic form $\omega_{B}$ induced by a symmetric nondegenerate bilinear Ad-invariant form $B$ on $\mathfrak{g}$.

(1) Let $\rho: \pi_{1}(M) \rightarrow G$ be such that $\rho r$ is good. Then the image of $T_{[\rho]} \mathcal{X}_{G}(M)$ in $T_{[\rho]} X_{G}(F)$ is Lagrangian.

(2) The non-singular part of $r^{*}\left(X_{G}(M)\right)$ in $X_{G}^{g}(F)$, denoted by us by $Y_{G}(M)$, is an isotropic submanifold of $X_{G}^{g}(F)$. (In particular, every connected component of $Y_{G}(M)$ of dimension $\frac{1}{2} \operatorname{dim} X_{G}(F)$ is Lagrangian.)

(3) If a connected component of $Y_{G}(M)$ contains an equivalence class of a reduced $G$-representation of $\pi_{1}(M)$, then it is a Lagrangian submanifold of $X_{G}(F)$.

Although we do not know any example of a 3-manifold $M$ such that $Y_{G}(M)$ is not Lagrangian, in light of the above theorem and the results of M. Kapovich mentioned in Section 12 we believe that such examples exist.

Here is an alternative version of Theorem 6.1(3):

Theorem 62. Let $X_{G}^{s}(M)$ be the set of equivalence classes of scheme smooth $G$ representations of $\pi_{1}(M)$. Then $X_{G}^{g}(F) \cap r^{*}\left(X_{G}^{s}(M)\right)$ is an immersed Lagrangian submanifold of $X_{G}^{g}(F)$.

Note that $X_{G}^{s}(M)$ may be empty, even if there exist good $G$-representations of $\pi_{1}(M)$. Note also that $r^{*}: X_{G}^{s}(M) \rightarrow X_{G}^{g}(F)$ does not have to be an immersion. Indeed, it is easy to choose an example of a 3-manifold $M$ satisfying the statement of Proposition 51 (2) all of whose $G$-representations are reduced and, hance, $X_{G}^{s}(M)$ has an arbitrarily large dimension. We prove versions of Theorems 61 and 62 for $F$ torus in Si5].

Theorems 61 and 62 have applications to Chern-Simons quantum field theory, $\mathrm{Ba}, \mathrm{Fr}, \mathrm{Gu}, \mathrm{JW}, \mathrm{We1}, \mathrm{We} 2, \mathrm{Si3}$. It is a $(2+1)$-topological quantum field theory, associating a Hilbert space $H(F)$ to every closed orientable surface $F$ and a vector $I(M) \in H(F)$ to every 3-manifold $M$ with $\partial M=F$. Although a mathematically rigorous version of this theory exists for compact groups $G$, [RT, BHMV], its constructions are combinatorial and algebraic in nature, yielding little information about the relations between $I(M)$ and the topology of $M$. Furthermore, a rigorous construction of this theory is still missing for complex algebraic groups.

One hopes to achieve a geometric construction of Chern-Simons TQFT for all groups through a procedure of geometric quantization which associates Hilbert spaces $H$ to symplectic manifolds $X$ and vectors in $H$ to Lagrangian subspaces of $X[\mathrm{Sn}, \mathrm{Wo}$. It is natural to expect that Witten's $I(M) \in H(F)$ is the vector associated with $Y_{G}(M)$. However, several obstacles exist in this approach, the first one being the question of whether $Y_{G}(M)$ is Lagrangian.

Theorems 61 and 62 are relevant also to Floer homology theory for 3-manifolds, cf. $\left[\mathrm{Cu}, \overline{\mathrm{BC} 1}, \overline{\mathrm{BC} 2}\right.$. If $F \subset M$ is a surface separating $M$ into $M_{1}$ and $M_{2}$ and $Y_{G}\left(M_{1}\right), Y_{G}\left(M_{2}\right)$ are Lagrangian submanifolds of $X_{G}(F)$, then one may consider Floer symplectic homology groups for such a splitting. (A difficulty with this approach lies in the non-compactness of $X_{G}(F)$.) 
For every representation $\rho: \pi_{1}(M) \rightarrow G$, the homomorphism $r: \pi_{1}(F) \rightarrow \pi_{1}(M)$ induces $r^{*}: H^{1}(M, A d \rho) \rightarrow H^{1}(F, A d \rho r)$. The proofs of Theorems 61 and 62 are based on the following:

Theorem 63. For every $\rho: \pi_{1}(M) \rightarrow G, r^{*} H^{1}(M, A d \rho)$ is a Lagrangian subspace of the symplectic space $\left(H^{1}(F, A d \rho r), \omega_{B}\right)$ with respect to every non-degenerate, Ad-invariant, symmetric, bilinear form $B$ on $\mathfrak{g}$.

In particular, for the trivial representation $\rho: \pi_{1}(M) \rightarrow \mathbb{C}^{*}$, Theorem 63 implies the following classical result:

Corollary 64. For every compact, orientable 3-manifold with a connected boundary $F$, the image of the map $r^{*}: H^{1}(M, \mathbb{C}) \rightarrow H^{1}(F, \mathbb{C})$ induced by the embedding $r: F \hookrightarrow M$ is a Lagrangian subspace of $H^{1}(F, \mathbb{C})$ with the symplectic form being the cup product.

Proof of Theorem 63. (1) We prove that

$$
\operatorname{dim} r^{*} H^{1}(M, A d \rho)=\frac{1}{2} \operatorname{dim} H^{1}(F, A d \rho r)
$$

first, by filling in the details of the approach of $[\mathrm{Fr}$. (This approach was communicated to us by Charlie Frohman.) By Poincaré-Lefschetz duality we have

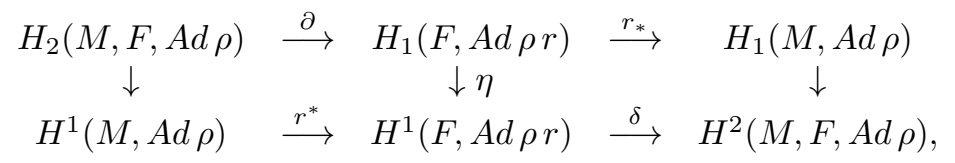

where all vertical maps are isomorphisms induced by Poincaré duality. By Corollary 59, the cap product

$$
H^{1}(F, A d \rho r) \times H_{1}(F, A d \rho r) \stackrel{\cap}{\longrightarrow} H_{0}(F, A d \rho r \otimes A d \rho r) \stackrel{B}{\longrightarrow} H_{0}(F, \mathbb{C})=\mathbb{C}
$$

is non-degenerate. Similarly,

$$
\begin{gathered}
H^{1}(M, A d \rho) \times H_{1}(M, A d \rho) \stackrel{\cap}{\longrightarrow} H_{0}(M, A d \rho \otimes A d \rho) \stackrel{B}{\longrightarrow} \mathbb{C}, \\
H^{2}(M, F, A d \rho) \times H_{2}(M, F, A d \rho) \stackrel{\cap}{\longrightarrow} H_{0}(M, F, A d \rho \otimes A d \rho) \stackrel{B}{\longrightarrow} \mathbb{C}
\end{gathered}
$$

are non-degenerate by Lemma 58 . Consider the isomorphisms

$$
\begin{aligned}
H^{1}(M, A d \rho) \simeq & \left(H_{1}(M, A d \rho)\right)^{*}, \quad H^{1}(F, A d \rho r) \simeq\left(H_{1}(F, A d \rho r)\right)^{*}, \\
& H^{2}(M, F, A d \rho) \simeq\left(H_{2}(M, F, A d \rho)\right)^{*}
\end{aligned}
$$

defined by these pairings. Under these identifications, $r^{*}$ and $r_{*}$ and $\partial$ and $\delta$ are the duals of each other. Hence

$$
\begin{gathered}
\operatorname{rank} r^{*}=\operatorname{rank} r_{*}=\operatorname{rank} \delta=\operatorname{dim} H^{1}(F, A d \rho r)-\operatorname{dim} K \operatorname{Ker} \delta \\
=\operatorname{dim} H^{1}(F, A d \rho r)-\operatorname{rank} r^{*} .
\end{gathered}
$$

(2) It remains to be proven that $r^{*} H^{1}(M, A d \rho)$ is an isotropic subspace of $H^{1}(F, A d \rho)$.

The pairing (27) identifies $H_{1}(F, A d \rho r)$ with $H^{1}(F, A d \rho r)^{*}$. The isomorphism $\eta^{-1}$ of (301) sends $\alpha \in H^{1}(F, A d \rho r)$ to $\eta^{-1}(\alpha) \in H_{1}(F, A d \rho r)$, which under the above identification is the functional $f_{\alpha}: H^{1}(F, A d \rho r) \rightarrow \mathbb{C}, f_{\alpha}(\beta)=\omega_{B}(\alpha, \beta)$.

By Lemma 58, the pairing

$$
H^{1}(M, A d \rho) \times H_{1}(M, A d \rho) \stackrel{\cap}{\longrightarrow} H_{0}(M, A d \rho \otimes A d \rho) \stackrel{B}{\longrightarrow} H_{0}(M, \mathbb{C})=\mathbb{C}
$$


is non-degenerate. If we use it to identify $H_{1}(M, A d \rho)$ with $H^{1}(M, A d \rho)^{*}$, then $r_{*}$ in (30) sends $f_{\alpha}$ to $f_{\alpha} r_{*}: H^{1}(M, A d \rho) \rightarrow \mathbb{C}$. By commutativity and exactness of (30), $f_{\alpha} r_{*}=0$ for every $\alpha \in r^{*}\left(H^{1}(M, A d \rho)\right)$. In other words, $f_{\alpha}(\beta)=0$ for every $\alpha, \beta \in r^{*}\left(H^{1}(M, A d \rho)\right)$.

Remark 65 . Let $G$ be reductive and $\rho: \pi_{1}(M) \rightarrow G$ be such that $\rho r: \pi_{1}(F) \rightarrow G$ is good. Then $\rho$ is good as well and, by Theorem $53(1)$ and (2), the following diagram commutes:

$$
\begin{array}{ccc}
H^{1}(M, A d \rho) & \stackrel{r^{*}}{\longrightarrow} & H^{1}(F, A d \rho r) \\
\downarrow \phi & & \downarrow \phi \\
T_{[\rho]} \mathcal{X}_{G}(M) & \stackrel{d r^{*}}{\longrightarrow} & T_{[\rho r]} \mathcal{X}_{G}(F),
\end{array}
$$

where $\phi$ is the isomorphism of Theorem $53(2)$.

Proof of Theorem 61. (1) is a direct consequence of Theorems 53, 63 and Remark 65.

(2) Since $Y_{G}(M)$ is smooth, it is enough to show the statement

$$
T_{[\rho r]} Y_{G}(M) \subset T_{[\rho r]} X_{G}(F) \quad \text { is isotropic }
$$

for a dense subset of points $[\rho r] \in Y_{G}(M)$ (in the complex topology). Let $X_{G}^{\prime}(M) \subset$ $X_{G}(M)$ be the non-singular part of $\left(r^{*}\right)^{-1} Y_{G}(M)$. Since $r^{*}\left(X_{G}^{\prime}(M)\right)$ is dense in $Y_{G}(F)$, it is enough to show that (32) holds for a dense subset of points in $r^{*}\left(X_{G}^{\prime}(M)\right)$. By Sard's theorem the there is a dense set of points in $[\rho r] \in r^{*}\left(X_{G}^{\prime}(M)\right)$ for which

$$
T_{[\rho r]} Y_{G}(M)=d r^{*} T_{[\rho]} X_{G}(M) .
$$

Therefore, it is enough to show that $d r^{*} T_{[\rho]} X_{G}(M)$ is isotropic in $T_{[\rho r]} X_{G}(F)$. This follows from (1) and the fact that $T_{[\rho]} X_{G}(M)$ is a subspace of $T_{[\rho]} \mathcal{X}_{G}(M)$.

(3) Let $\rho: \pi_{1}(M) \rightarrow G$ be a reduced, irreducible representation whose conjugacy class belongs to $Y_{G}(M)$. By Theorem 53(2), both $\phi$ 's in the diagram of Remark 65 are isomorphisms. By Theorem [63, $\operatorname{dim} T_{[\rho]} C=\frac{1}{2} \operatorname{dim} T_{[\rho]} X_{G}(F)$. Since $C$ and $X_{G}^{g}(F)$ are smooth, that equality holds for all points of $C$. Now the statement follows from (2).

Proof of Theorem 62. By Proposition 33, $X_{G}^{g}(F) \subset X_{G}(F)$ is open and, hence, $U=\left(r^{*}\right)^{-1}\left(X_{G}^{g}(F)\right) \cap X_{G}^{s}(M)$ is an open subset of $X_{G}^{s}(M)$. Since $\phi$ 's in the diagram of Remark 65 are isomorphisms, by Theorem 63.

$$
d r^{*}: T_{[\rho]} U \rightarrow T_{[\rho]} X_{G}^{g}(F)
$$

has constant rank for all $[\rho]$ in $U$. By the Constant Rank Theorem, for every $[\rho] \in U$ there is a neighborhood $V$ of $[\rho]$ in $U$ and a neighborhood $W$ of $r^{*}([\rho])$ in $X_{G}^{g}(G)$ such that $r^{*}(V) \cap W$ a submanifold of $W$. Consequently, $r^{*}(U)=r^{*}\left(X_{G}^{s}(M)\right) \cap X_{G}^{g}(F)$ is an immersed submanifold of $X_{G}^{g}(F)$.

\section{REFERENCES}

[AM] S. Akbulut and J. McCarthy, Casson's invariant for oriented homology 3-spheres an exposition, Mathematical Notes, 36. Princeton University Press, Princeton, NJ, 1990. MR 1030042 (90k:57017)

[At] M. Atiyah, Geometry and Physics of Knots, Cambridge University Press, 1990. MR.1078014 (92b:57008)

[AB] M. Atiyah and R. Bott, The Yang-Mills equations over Riemann surfaces, Philos. Trans. Roy. Soc. London Ser. A 308 (1983) 523-615. MR702806 (85k:14006) 
[AP] S. Abeasis and M. Pittaluga, On a minimal set of generators for the invariants of $3 \times 3$ matrices, Comm. in Alg. 17 (1989), no. 2, 487-499. MR.978487 (90d:15021)

[AMW] A. Alekseev, E. Meinrenken and C. Woodward, Duistermaat-Heckman measures and moduli spaces of flat bundles over surfaces. Geom. Funct. Anal. 12 (2002), no. 1, 1-31. MR.1904554 (2003d:53151)

[Au] M. Audin, Lectures on gauge theory and integrable systems, in Gauge theory and symplectic geometry (Montreal, PQ, 1995), 1-48, NATO Adv. Sci. Inst. Ser. C Math. Phys. Sci., 488, Kluwer Acad. Publ., Dordrecht, 1997. MR1461568 (99f:58028)

[Ba] S. Baseilhac, Chern Simons theory in dimension three, www-fourier.ujf-grenoble.fr/ 〜baseilha

[Be] L. Ben Abdelghani, Variéte des caractères et slice étale de l'espace des représentations d'un group, Ann. de la faculté des sciences de Toulouse, 11 (2002) no. 6, 19-32. MR $1986380(2004 \mathrm{~h}: 14067)$

[BC1] H. U. Boden and C. L. Curtis, Splicing and the $S L_{2}(\mathbb{C})$ Casson invariant, Proc. Amer. Math. Soc. 136 (2008), no. 7, 2615-2623, arXiv: 0707.4134. MR2390534(2009c:57022)

[BC2] H. U. Boden and C. L. Curtis, The $S L_{2}(C)$ Casson invariant for Seifert fibered homology spheres and surgeries on twist knots, J. Knot Theory Ramifications 15 (2006), no. 7, 813-837. MR2264157 (2007g:57017)

[BHe] H. Boden and C. Herald, The $S U(3)$ Casson invariant for integral homology 3-spheres, J. Differential Geom. 50 (1998), 147-206. MR.1678493(2000d:57011)

[BK1] V. V. Benyash-Krivets, The transcendence basis of the field of functions of the variety of characters of a free group, Dokl. Akad. Nauk BSSR 33 (1989), no. 4, 297-301. MR.1000642 (90j:20026)

[BK2] V. V. Benyash-Krivets, Varieties of two-dimensional characters of groups, and amalgamated products, Dokl. Nats. Akad. Nauk Belarusi 44 (2000), no. 1, 5-7. MR.1776637 (2001d:20007)

[BK3] V. V. Benyash-Krivets, Character varieties of finitely generated groups, Vestsi Nats. Akad. Navuk Belarusi Ser. Fiz.-Mat. Navuk, (2002), no. 1, 19-24, MR1952806 (2003h:20015)

[BKCh] V. V. Benyash-Krivets and V. I. Chernousov, Varieties of representations of fundamental groups of compact nonoriented surfaces, Mat. Sb. 188 (1997), no. 7, 47-92; translation in Sb. Math. 188 (1997), no. 7, 997-1039. MR.1474855 (98j:57002)

[BHMV] C. Blanchet, N. Habegger and G. Masbaum, and P. Vogel. Topological quantum field theories derived from the Kauffman bracket, Topology, 34(4) (1995), 883-927. MR.1362791 (96i:57015)

[BF] H. U. Boden and S. Friedl, Metabelian SL(n,C) representations of knot groups, Pacific J. Math. 238 (2008), no. 1, 7-25. MR2443505 (2010c:57007)

[BB] M. Boileau and S. Boyer, On character varieties, sets of discrete characters, and nonzero degree maps, arXiv.org: 0701384

[Bo] A. Borel, Linear Algebraic Groups, 2nd enlarged ed., Springer 1991. MR.1102012 (92d:20001)

[BLZ] S. Boyer, E. Luft and X. Zhang, On the algebraic components of the SL $(2, \mathbb{C})$ character varieties of knot exteriors, Topology 41 (2002), no. 4, 667-694. MR1905834 (2003g:57030)

[BMR] M. Bate, B. Martin and G. Röhrle, A geometric approach to complete reducibility, Invent. Math. 161 (2005), 177-218. MR2178661(2007k:20101)

[BN] S. Boyer and A. Nicas, Varieties of group representations and Casson's invariant for rational homology 3-spheres, Trans. Amer. Math. Soc. 322 (1990), 507-522. MR972701 (92a:57020)

[BZ1] S. Boyer and X. Zhang, Finite Dehn surgery on knots, J. Amer. Math. Soc. 9 (1996), 1005-1050. MR.1333293 (97h:57013)

[BZ2] S. Boyer and X. Zhang, On Culler-Shalen seminorms and Dehn filling, Annals of Math. 148 (1998), 737-801. MR.1670053(2000d:57028)

[BZ3] S. Boyer and X. Zhang, On simple points of character varieties of 3-manifolds, Knots in Hellas '98 (Delphi), 27-35, Ser. Knots Everything, 24, World Sci. Publ., 2000. MR.1865698 (2002i:57027)

[Br] K. S. Brown, Cohomology of groups, Graduate Texts in Mathematics, Springer, 1982. MR672956 (83k:20002) 
[BCo] S. Bratholdt and D. Cooper, On the topology of the character variety of a free group, Dedicated to the memory of Marco Reni, Rend. Instit. Mat. Univ. Trieste 32 (2001), suppl. 1, 45-53 (2002). MR1889465(2003d:14072)

[BH] G. W. Brumfiel and H. M. Hilden, $S l(2)$ Representations of Finitely Presented Groups, Contemp. Math. 187 (1995). MR1339764(96g:20004)

[Bu] D. Bullock, Rings of $S L_{2}(\mathbb{C})$-characters and the Kauffman bracket skein module, Comment. Math. Helv. 72 (1997), 521-542. MR1600138 (98k:57008)

[BIW] M. Burger, A. Iozzi and A. Wienhard, Surface group representations with maximal Toledo invariant, Ann. of Math. 172 (2010), no.1, 517-566. MR1979350(2004e:53076)

[CHM] M. A. de Cataldo, T. Hausel and L. Migliorini, Topology of Hitchin systems and Hodge theory of character varieties, arXiv: 1004.1420

[CM] L. Charles and J. Marché, Multicurves and regular functions on the representation variety of a surface in $S U(2)$, arXiv: 0901.3064

[CL] D. Cooper and D. Long, Representation theory and the A-polynomial of a knot, Chaos Solitons Fractals 9 (1998), 749-763. MR1628754(99c:57013)

[CCGLS] D. Cooper, M. Culler, H. Gillet, D.D. Long and P. B. Shalen, Plane Curves Associated to Character Varieties of 3-manifolds, Inventiones Math. 118 (1994), 47-84. MR 1288467 (95g:57029)

[CLO] D. Cox, J. Little and D. O'Shea, Ideals, Varieties, and Algorithms, An introduction to Computational Algebraic Geometry and Commutative Algebra, 2nd edition, Springer 1992. MR1189133 (93j:13031)

[CS] M. Culler and P. B. Shalen, Varieties of group representations and splittings of 3manifolds, Ann. of Math. 117 (1983), 109-146. MR683804 (84k:57005)

[Cu] C. L. Curtis, An intersection theory count of the SL2(C)-representations of the fundamental group of a 3-manifold, Topology 40 (2001), 773-787. MR.1851563(2002k:57022)

[Da] G. Daskalopoulos, The topology of the space of stable bundles on a compact Riemann surface, J. Differential Geom. 36 (3) (1992) 699-746. MR.1189501(93i:58026)

[DDW] G. Daskalopoulos, S. Dostoglou and R. Wentworth, On the Morgan-Shalen compactification of the $\mathrm{SL}(2, C)$ character varieties of surface groups, Duke Math. J. 101 (2000), no. 2, 189-207. MR 1738182 (2000m:32024)

[DWWW] G. Daskalopoulos, J. Weitsman, R. Wentworth and G. Wilkin, Morse Theory and Hyper-Kahler Kirwan Surjectivity for Higgs Bundles, arXiv:math/0701560.

[DWW] G. D. Daskalopoulos, R. A. Wentworth and G. Wilkin, Cohomology of $S L(2, C)$ character varieties of surface groups and the action of the Torelli group, arXiv:0808.0131.

[Do] I. Dolgachev, Introduction to geometric invariant theory, Notes of the Series of Lectures held at the Seoul National University, 1994. MR.1312159 (96a:14019)

[Du] N. Dunfield, Cyclic surgery, degrees of maps of character curves, and volume rigidity for hyperbolic manifolds, Invent. Math. 136 (1999), 623-657. MR1695208 (2000d:57022)

[DG] N. M. Dunfield, S. Garoufalidis, Nontriviality of the A-polynomial for knots in $S^{3}$, Algebr. Geom. Topol. 4 (2004), 1145-1153. MR2113900(2005i:57004)

$[\mathrm{EH}] \quad$ D. Eisenbud and J. Harris, The geometry of schemes, Graduate Texts in Mathematics, Springer 2000. MR 1730819 (2001d:14002)

[FL1] C. Florentino and S. Lawton, The topology of moduli spaces of free group representations. MR2529483 (2010h:14075)

[FL2] C. Florentino and S. Lawton, Singularities of free group character varieties, arXiv:0907. 4720

[Fo] J. Fogarty, Invariant Theory, W. A. Benjamin, Inc. 1969. MR0240104 (39:1458)

[Fr] D. S. Freed, Classical Chern-Simons Theory, 1, Adv. in Math. 113 (1995), 2371920-303. MR:1337109 (96h:58019)

[FG] C. Frohman and R. Gelca, Skein modules and the noncommutative torus, Trans. Amer. Math. Soc. 352 (2000), 4877-4888. MR1675190 (2001b:57014)

[FGL] C. Frohman, R. Gelca and W. LoFaro, The A-polynomial from the noncommutative viewpoint, Trans. Amer. Math. Soc. 354 (2001), 735-747. MR1862565 (2003a:57020)

$[\mathrm{FH}] \quad$ W. Fulton and J. Harris, Representation Theory, A First Course, Graduate Texts in Mathematics, Springer, 1991. MR 1153249 (93a:20069)

[GGM] O. Garcia-Prada, P. B. Gothen and I. Mundet i Riera, Higgs bundles and surface group representations in the real symplectic group, arXiv:0809.0576. 
[Ga] S. Garoufalidis, On the characteristic and deformation varieties of a knot, Proceedings of the Casson Fest, 291-309, Geom. Topol. Monogr., 7, Geom. Topol. Publ., Coventry, 2004. MR2172488 (2006j:57028)

[Ge] R. Gelca, On the relation between the $A$-polynomial and the Jones polynomial, Proc. Amer. Math. Soc. 130 (2002), no. 4, 1235-1241. MR.1873802 (2002m:57015)

[Go1] W. M. Goldman, Representations of fundamental groups of surfaces, Geometry and topology (College Park, Md., 1983/84), 95-117, Lecture Notes in Math., 1167, Springer, Berlin, 1985. MR827264 (87j:32068)

[Go2] W. Goldman, The symplectic nature of fundamental groups of surfaces, Adv. in Math. 54 (1984), 200-225 MR762512 (86i:32042)

[Go3] W. M. Goldman, Geometric structures on manifolds and varieties of representations. Geometry of group representations, (Boulder, CO, 1987), 169-198, Contemp. Math., 74, Amer. Math. Soc., 1988. MR957518 (90i:57024)

[Go4] W. M. Goldman, Invariant functions on Lie groups and Hamiltonian flows of surface group representations, Invent. Math. 85 (1986), no. 2, 263-302. MR.846929 (87j:32069)

[Go5] W. M. Goldman, Topological components of spaces of representations, Invent. Math. 93 (1988), no. 3, 557-607. MR952283 (89m:57001)

[Go6] W. Goldman, Action of the modular group on real $S L(2)$-characters of a one-holed torus, Geom. and Top. 7 (2003), 443-486. MR2026539(2004k:57001)

[Go7] W. M. Goldman, The complex-symplectic geometry of $\operatorname{SL}(2, \mathbb{C})$-characters over surfaces. Algebraic groups and arithmetic, 375-407, Tata Inst. Fund. Res., Mumbai, 2004. MR 2094117 (2005i:53110)

[Go8] W. Goldman, An ergodic action of the outer automorphism group of a free group, Geom. Funct. Anal.17 (2007), no. 3, 793-805. MR2346275 (2008g:57001)

[Go9] W. Goldman, Trace coordinates on Fricke spaces of some simple hyperbolic surfaces, preprint.

[GM1] W. M. Goldman and J. J. Millson, The deformation theory of representations of fundamental groups of compact Kähler manifolds, Bull. Amer. Math. Soc. (N.S.) 18 (1988), no. 2, 153-158. MR.929091 (89f:53100)

[GM2] W. Goldman and J. Millson, The deformation theory of representations of fundamental groups of Kähler manifolds, Publ. Math. d'I. H. E. S., 67 (1988), 43-96. MR972343 (90b:32041)

[GM] F. González-Acuña and J. M. Montesinos-Amilibia, On the character variety of group representations in $\operatorname{SL}(2, C)$ and $\operatorname{PSL}(2, C)$, Math. Z. 214 (1993), no. 4, 627-652. MR 1248117 (94k:57022)

[GO] V. V. Gorbatsevich and A. L. Onishchik, Lie Transformation Groups, Encyclopaedia of Mathematical Sciences, Vol. 20, Editor: A.L. Onishchik, Springer, 1993. MR 1306739

[GW] O. Guichard and A. Wienhard, Topological Invariants of Anosov Representations, J. of Topology, to appear, arXiv:0907.0273v2.

[Gu] S. Gukov, Three-dimensional quantum gravity, Chern-Simons theory, and the A-polynomial. Comm. Math. Phys. 255 (2005), no. 3, 577-627. MR2134725 (2006f:58029)

[Ha] R. Hartshorne, Algebraic Geometry, Graduate Texts in Mathematics, Springer, 1977. MR0463157 (57:3116)

[HLR] T. Hausel, E. Letellier and F. Rodriguez-Villegas, Topology of character varieties and representations of quivers. C. R. Math. Acad. Sci. Paris 348 (2010), no. 3-4, 131-135. MR.2600063

[HT] T. Hausel and M. Thaddeus, Relations in the cohomology ring of the moduli space of rank 2 Higgs bundles. J. Amer. Math. Soc. 16 (2003), no. 2, 303-327. MR1949162 (2004b:14055)

[HP1] M. Heusener and J. Porti, Deformations of reducible representations of 3-manifold groups into $P S L(2, \mathbb{C})$, Alg. Geom. Top 5 (2005), 965-997. MR2171800(2006e:57016)

[HP2] M. Heusener and J. Porti, The variety of characters in $\mathrm{PSL}_{2}(\mathbb{C})$, Bol. Soc. Mat. Mexicana 10 (2004), no. 3, Special Issue, 221-237. MR2199350 (2006m:57020)

[HLM1] H. M. Hilden, M. T. Lozano and J. M. Montesinos-Amilibia, On the character variety of tunnel number 1 knots, J. London Math. Soc. (2) 62 (2000), no. 3, 938-950. MR.1794296 (2001i:57009) 
[HLM2] H. M. Hilden, M. T. Lozano and J. M. Amilibia-Montesinos, Character varieties and peripheral polynomials of a class of knots, J. Knot Theory Ramifications 12 (2003), no. 8, 1093-1130. MR2017984(2004i:57005)

[Hi1] N. J. Hitchin, The self-duality equations on a Riemann surface, Proc. London Math. Soc. (3) 55 (1987), 59-126. MR887284 (89a:32021)

[Hi2] N. J. Hitchin, Lie groups and Teichmüller space, Topology 31 (1992), 449-473. MR 1174252 (93e:32023)

[Ho1] R. Horowitz, Characters of free groups represented in the two-dimensional special linear group, Comm. Pure Appl. Math. 25 (1972), 635-649. MR0314993 (47:3542)

[Ho2] R. Horowitz, Induced automorphisms on Fricke characters on free groups, Trans. of AMS 208 (1975), 41-50. MR0369540 (51:5773)

[HS] J. Hoste and P. Shanahan, A formula for the A-polynomial of twist knots, J. Knot Theory Ramifications, 13 (2004), no. 2, 193-209. MR2047468 (2005c:57006)

[Hu] J. E. Humphreys, Linear Algebraic Groups, Graduate Texts in mathematics, Springer, 1975. MR0396773 (53:633)

[Je1] L. C. Jeffrey, Symplectic forms on moduli spaces of flat connections on 2-manifolds, Geometric topology (Athens, GA, 1993), 268-281, AMS/IP Stud. Adv. Math., 2.1, Amer. Math. Soc., Providence, RI, 1997. MR1470732 (99b:58043)

[Je2] L. Jeffrey, Group cohomology construction of the cohomology of moduli spaces of flat connections on 2 manifolds, Duke Math. J. 77 (1995), 407-429. MR1321064 (96m:58029)

[Je3] L. C. Jeffrey, Flat connections on oriented 2-manifolds, Bull. London Math. Soc. 37 (2005), 1-14. MR2105813 (2005i:53111)

[JK] L. C. Jeffrey and F. C. Kirwan, Intersection theory on moduli spaces of holomorphic bundles of arbitrary rank on a Riemann surface, Ann. of Math. (2) 148 (1998), no. 1, 109-196. MR1652987 (2000c:14045)

[JW] L. C. Jeffrey and J. Weitsman, Half density quantization of the moduli space of flat connections and Witten's semiclassical manifold invariants, Topology 32(3) 1993, 509529. MR1231958 (95f:58038)

[JM] D. Johnson and J. J. Millson, Deformation Spaces Associated to Compact Hyperbolic Manifolds, in "Discrete Groups in Geometry and Analysis", Proceedings of a Conference Held at Yale Univeristy in Honor of G.D. Mostow on his Sixtieth Birthday.

[Ka1] M. Kapovich, lecture at CRM, Montreal 2001.

[Ka2] M. Kapovich, private communication.

[KM] M. Kapovich and J. J. Millson, On representation varieties of Artin groups, projective arrangements and the fundamental groups of smooth complex algebraic varieties, Inst. Hautes Études Sci. Publ. Math. 88 (1998), 5-95 (1999). MR.1733326 (2001d:14024)

[KK] P. Kirk and E. Klassen, Chern-Simons invariants of 3-manifolds decomposed along tori and the circle bundle over the representation space of $T^{2}$, Comm. Math. Phys. 153 (1993) no. 3, 521-557. MR1218931 (94d:57042)

[KrM] P. Kronheimer and T. Mrowka, Dehn surgery, the fundamental group and SU(2), Math. Res. Lett. 11 (2004), no. 5-6, 741-754. MR2106239 (2005k:57018)

$[\mathrm{KN}] \quad$ A. D. King and P. E. Newstead, On the cohomology ring of the moduli space of rank 2 vector bundles on a curve, Topology 37 (1998) 407-418. MR.1489212 (99a:14019)

[Ki] F. C. Kirwan, The cohomology rings of moduli spaces of bundles over Riemann surfaces, J. Amer. Math. Soc. 5 (1992) 853-906. MR.1145826 (93g:14016)

[La1] S. Lawton, Generators, Relations and Symmetries in Pairs of 3x3 Unimodular Matrices, J. Algebra 313 (2007), no. 2, 782-801. MR2329569(2008k:16039)

[La2] S. Lawton, Poisson geometry of $S L(3, \mathbb{C})$-character varieties relative to a surface with boundary, Trans. Amer. Math. Soc. 361 (2009), 2397-2429. MR2471924(2009k:53224)

[La3] S. Lawton, Minimal Affine Coordinates for $S L(3, \mathbb{C})$ Character Varieties of Free Groups, J. Algebra 320 (2008), no. 10, 3773-3810. MR2457722(2009j:20060)

[La4] S. Lawton, Obtaining the One-Holed Torus from Pants: Duality in an SL(3,C)Character Variety, Pacific J. Math. 242 (2009), no. 1, 131-142. MR2525506 (2010g:53164)

[La5] S. Lawton, Algebraic Independence in SL(3,C) Character Varieties of Free Groups, aXiv:0807.0798 
[LP] S. Lawton and E. Peterson, Spin networks and SL(2,C)-Character varieties, Eur. Math. Soc. Zurich, 2009. MR2516745

[Le1] T. Q. Le, Varieties of representations and their subvarieties of cohomology jumps for knot groups. (Russian) Mat. Sb. 184 (1993), no. 2, 57-82; translation in Russian Acad. Sci. Sb. Math. 78 (1994), no. 1, 187-209. MR.1214944 (94a:57016)

[Le2] T. Q. Le, The colored Jones polynomial and the A-polynomial of knots, Adv. Math. 207 (2006), no. 2, 782-804. MR2271986 (2007k:57021)

[Li] J. Li, The space of surface group representations, Manuscripta Math. 78 (1993) 223243. MR.1206154 (94c:58022)

[LR1] D. D. Long and A. W. Reid, Commensurability and the character variety, Math. Res. Lett. 6 (1999), no. 5-6, 581-591. MR1739217 (2000m:57017)

[LR2] D. D. Long and A. W. Reid, Integral points on character varieties, Math. Ann. 325 (2003), no. 2, 299-321. MR1962051 (2004c:57028)

[LM] A. Lubotzky and A. Magid, Varieties of representations of finitely generated groups, Memoirs of the AMS, 336 (1985). MR818915 (87c:20021)

[Lu] D. Luna, Slices étale, Bull. Soc. Math. Fr., Suppl. Mèm. 33 (1973), 81-105. MR.0342523 (49:7269)

[Me] E. Meinrenken, Witten's formulas for intersection pairings on moduli spaces of flat G-bundles, Adv. in Math. 197 (2005), 140-197. MR2166180(2006d:53108)

[MW] E. Meinrenken and C. Woodward, Moduli spaces of flat connections on 2-manifolds, cobordism, and Witten's volume formulas.

[MS] J. W. Morgan and P. B. Shalen, Valuations, trees, and degenerations of hyperbolic structures. I, Ann. of Math. (2) 120 (1984), 401-476. MR769158 (86f:57011)

[Mo] K. Motegi, Haken manifolds and representations of their fundamental groups in SL(2,C), Topology Appl. 29 (1988), no. 3, 207-212. MR953952 (89h:57010)

[MFK] D. Mumford, J. Fogarty and F. Kirwan, Geometric Invariant Theory, Springer-Verlag 1994. MR:1304906 (95m:14012)

[Na] K. Nakamoto, Representation varieties and character varieties, Publ. Res. Inst. Math. Sci. 36 (2000), no. 2, 159-189. MR.1753200 (2001f:14026)

[NS] M. S. Narasimhan and C. S. Seshadri, Stable and unitary vector bundles on a compact Riemann surface, Ann. Math. 82 (1965), 540-567. MR0184252 (32:1725)

[Ne] P. E. Newstead, Introduction to Moduli Problems and Orbit Spaces, Tata Institute Lecture Notes, Springer 1978. MR546290 (81k:14002)

[Ol] A. G. Oliveira, Representations of surface groups in the projective general linear group, Int. J. of Math., in press.

$[\mathrm{PX}] \quad$ D. Pickrell and E. Xia, Ergodicity of mapping class group actions on representation varieties. I. Closed surfaces, Comment. Math. Helv. 77 (2002), no. 2, 339-362. MR 1915045 (2003i:22025)

[PBK] V.P. Platonov and V. V. Benyash-Krivets, Character rings of representations of finitely generated groups, (Russian) Translated in Proc. Steklov Inst. Math. 1991, no. 4, 203213, Galois theory, rings, algebraic groups and their applications (Russian) Trudy Mat. Inst. Steklov.183 (1990), 169-178, 227. MR.1092029 (91m:20064)

[PV] V. L. Popov and E. B. Vinberg, Invariant Theory, in Encyclopaedia of Mathematical Sciences, Algebraic Geometry IV, A. N. Parshin, I. R. Shafarevich, eds., Springer 1994. MR.1309681 (95g:14002)

[Po] J. Porti, Torsion de Reidemeister pour les Variétés Hyperboliques, Memoirs of A.M.S. 612, vol. 128, (1997). MR1396960 (98g:57034)

[PS1] J. H. Przytycki and A. S. Sikora, On Skein Algebra of a Group, in "Knot theory," Banach Center Publications 42, Warsaw, 1998, 297-306. MR1634463 (99e:57019)

[PS2] J. H. Przytycki and A. S. Sikora, On Skein Algebras and $S l_{2}(\mathbb{C})$-Character Varieties, Topology 39 (2000), no. 1, 115-148. MR1710996 (2000g:57026)

[Rac] S. Racanière, Kirwan Map and Moduli Space of Flat Connections, Mathematical Research Letters 11 (2004), 419-433. MR2092897 (2005h:53150)

[Rag] M. S. Raghunatan, Discrete subgroups of Lie groups, Ergebnisse der Mathematik und ihrer Grenzgebiete, Band 68, Springer-Verlag, 1968. MR0507234 (58:22394a)

[Ra] A. S. Rapinchuk, On SS-rigid groups and A. Weil's criterion for local rigidity. I, Manuscripta Math. 97 (1998), no. 4, 529-543. MR1660132 (99m:20019) 
[RBK] A. S. Rapinchuk and V. V. Benyash-Krivets, Geometric theory of representations for fundamental groups of compact oriented surfaces, Dokl. Akad. Nauk 329 (1993), no. 2, 140-143; translation in Russian Acad. Sci. Dokl. Math. 47 (1993), no. 2, 211-215. MR.1228102 (94i:20070)

[RBC] A. S. Rapinchuk, V. V. Benash-Krivetz and V. I. Chernousov, Representation Varieties of the fundamental groups of compact orientable surfaces, Israel J. of Math. 931996 29-71. MR.1380633 (98a:57002)

[RT] N. Yu. Reshetikhin and V. G. Turaev, Ribbon graphs and their invariants derived from quantum groups, Comm. Math. Phys. 127 (1990), no. 1, 1-26. MR.1036112 (91c:57016)

[Ri1] R. W. Richardson, Commuting varieties of semi-simple Lie algebras and algebraic groups, Compositio Math. 38 (1979), no. 3, 311-327. MR535074 (80c:17009)

[Ri2] R. W. Richardson, Conjugacy classes of $n$-tuples in Lie algebras and algebraic groups, Duke Math. J. 57 (1988), no. 1, 1-35. MR952224(89h:20061)

[Ril1] R. Riley, Parabolic representations of knot groups, I, Proc. London Math. Soc. 3(24) (1972), 217-242. MR0300267 (45:9313)

[Ril2] R. Riley, Nonabelian representations of 2-bridge knot groups, Quart. J. Math. Oxford 235 (1984), 191-208. MR.745421 (85i:20043)

[Sa] K. Saito, Character variety of representations of a finitely generated group in $\mathrm{SL}_{2}$, Topology and Teichmüller spaces (Katinkulta, 1995), 253-264, World Sci. Publ., 1996. MR 1659663 (99k:20011)

[Se] J.-P. Serre, Complète Réductibilité. Séminaire Bourbaki, no 932, 56ème année, 20032004. MR2167207 (2006d:20084)

[Shf] I. R. Shafarevich, Basic Algebraic Geometry I, Springer, 1995. MR0447223 (56:5538)

[Shl] P. Shalen, Representations of 3-manifold groups, in Handbook of Geometric Topology, R. Daverman and R. Sher, eds. North-Holland, Amsterdam, 2002, 955-1044. MR 1886685 (2003d:57002)

[Si1] A. S. Sikora, $S L_{n}$-character varieties as spaces of graphs, Trans. A.M.S. 353 (2001), no. 7, 2773-2804. MR.1828473(2003b:57004)

[Si2] A. S. Sikora, Skein theory for SU(n)-quantum invariants, Algebr. Geom. Topol. 5 (2005), 865-897. MR2171796 (2006j:57033)

[Si3] A. S. Sikora, Quantizations of character varieties and quantum knot invariants, Algebr. Geom. Topol. to appear, arXiv:0807.0943

[Si4] A. S. Sikora, Algebraic description of character varieties, preprint.

[Si5] A. S. Sikora, Character varieties of abelian groups, in preparation.

[Sim1] C. Simpson, Moduli of representations of the fundamental group of a smooth projective variety. I. Inst. Hautes Études Sci. Publ. Math. 79 (1994), 47-129. MR1307297 (96e:14012)

[Sim2] C. Simpson, Moduli of representations of the fundamental group of a smooth projective variety. II. Inst. Hautes Études Sci. Publ. Math. 80 (1994), 5-79 (1995). MR1320603 (96e:14013)

[Sn] J. Sniatycki, Geometric Quantization and Quantum Mechanics, Springer, 1980. MR.554085 (82b:81001)

[Sp] E. Spanier, Singular homology and cohomology with local coefficients and duality for manifolds, Pacific J. Math. 160 (1993), no. 1, 165-200. MR1227511 (94h:55009)

[SS] J. Souto and P. Storm, Dynamics of the mapping class group action on the variety of $\mathrm{PSL}_{2} \mathbb{C}$ characters, Geom. Topol. 10 (2006), 715-736. MR2240903 (2007k:57035)

[St] R. Steinberg, Regular elements of semisimple algebraic groups, Inst. Hautes Etudes Sci. Publ. Math. 25 (1965), 49-80. MR0180554(31:4788)

[Th1] M. Thaddeus, Conformal field theory and the cohomology of the moduli space of stable bundles, J. Differential Geom. 35 (1992) 131-149. MR1152228 (93g:14017)

[Th2] M. Thaddeus, Stable pairs, linear systems and the Verlinde formula, Invent. Math. 117 (1994), 317-353. MR 1273268 (95e:14006)

[Th3] M. Thaddeus, A perfect Morse function on the moduli space of flat connections, Topology 39 (2000), no. 4, 773-787. MR:1760428(2001h:53123)

[Ti] S. Tillmann, Character varieties of mutative 3-manifolds, Algebr. Geom. Topol. 4 (2004), 133-149. MR2059186(2005c:57016)

[Th] W. P. Thurston, Three-dimensional geometry and topology, Volume 1, Edited by Silvio Levy, Princeton Univeersity Press, 1997. MR1435975 (97m:57016) 
[Wa] W. Waterhouse, Introduction to Affine Group Schemes, Graduate Texts in Mathematics, Springer, 1997. MR547117 (82e:14003)

[Wb] C. A. Weibel, Introduction to Homological Algebra, Cambridge Univ. Press, 1994. MR:1269324 (95f:18001)

[Wei] A. Weil, Remarks on cohomology of groups, Ann. of Math. 80 (1964) (1) 149-157. MR 0169956 (30:199)

[We1] J. Weitsman, Quantization via real polarization of the moduli space of flat connections and Chern-Simons gauge theory in genus 1, Commun. Math. Phys. 137 (1991), 175190. MR1099261 (92f:58071)

[We2] J. Weitsman, Real polarization of the moduli space of flat connections on a Riemann surface, Commun. Math. Phys. 145 (1992), 425-433. MR1162354 (93e:58077)

[We3] J. Weitsman, Geometry of the intersection ring of the moduli space of flat connections and the conjectures of Newstead and Witten, Topology 37(1) (1998) 115-132. MR:1480881 (99m:57030)

[We] R. A. Wentworth, The action of the mapping class group on representation varieties, preprint.

[Wi1] E. Witten, Quantum field theory and the Jones polynomial, Commun. Math. Phys. 121 (1989), no. 3, 351-399. MR990772 (90h:57009)

[Wi2] E. Witten, On quantum gauge theories in two dimensions, Commun. Math. Phys. 141 (1991) 153-209. MR 1133264 (93i:58164)

[Wi3] E. Witten, Two-dimensional gauge theories revisited, J. Geom. Phys. 9 (1992) 303-368. MR 1185834 (93m:58017)

[Wh] A. Whittemore, On special linear characters of free groups of rank $n \geq 4$, Proc. of A.M.S. 40 (1973), no. 2, 383-388. MR0322064(48:428)

[Wi] A. Wienhard, The action of the mapping class group on maximal representations, Geom. Dedicata 120 (2006), 179-191. MR2252900 (2008g:20112)

[Wol] S. A. Wolpert, The Weil-Petersson metric geometry, in Handbook of Teichmueller theory, Vol. II, IRMA Lectures, European Math. Soc., 2009. MR2497791|(2010i:32012)

[Wo] N. M. J. Woodhouse, Geometric Quantization, 2nd ed., Clarendon Press, Oxford, 1992. MR.1183739 (94a:58082)

[Za] D. Zagier, On the cohomology of moduli spaces of rank two vector bundles over curves, in The moduli space of curves (Texel Island, 1994), 533-563, Progr. Math., 129, Birkhäuser, 1995. MR1363070 (97g:14010)

Department of Mathematics, 244 Math. Bldg., University at Buffalo, SUny, BufFALO, NEW YORK 14260

E-mail address: asikora@buffalo.edu 